\title{
Low-level light therapy of the eye and brain
}

This article was published in the following Dove Press journal:

Eye and Brain

14 October 2011

Number of times this article has been viewed

\author{
Julio C Rojas ${ }^{1,2}$ \\ F Gonzalez-Lima' \\ 'Departments of Psychology, \\ Pharmacology and Toxicology, \\ University of Texas at Austin, Austin, \\ TX; ${ }^{2}$ Department of Neurology and \\ Neurotherapeutics, University of \\ Texas Southwestern Medical Center, \\ Dallas, TX, USA
}

\begin{abstract}
Low-level light therapy (LLLT) using red to near-infrared light energy has gained attention in recent years as a new scientific approach with therapeutic applications in ophthalmology, neurology, and psychiatry. The ongoing therapeutic revolution spearheaded by LLLT is largely propelled by progress in the basic science fields of photobiology and bioenergetics. This paper describes the mechanisms of action of LLLT at the molecular, cellular, and nervous tissue levels. Photoneuromodulation of cytochrome oxidase activity is the most important primary mechanism of action of LLLT. Cytochrome oxidase is the primary photoacceptor of light in the red to near-infrared region of the electromagnetic spectrum. It is also a key mitochondrial enzyme for cellular bioenergetics, especially for nerve cells in the retina and the brain. Evidence shows that LLLT can secondarily enhance neural metabolism by regulating mitochondrial function, intraneuronal signaling systems, and redox states. Current knowledge about LLLT dosimetry relevant for its hormetic effects on nervous tissue, including noninvasive in vivo retinal and transcranial effects, is also presented. Recent research is reviewed that supports LLLT potential benefits in retinal disease, stroke, neurotrauma, neurodegeneration, and memory and mood disorders. Since mitochondrial dysfunction plays a key role in neurodegeneration, LLLT has potential significant applications against retinal and brain damage by counteracting the consequences of mitochondrial failure. Upon transcranial delivery in vivo, LLLT induces brain metabolic and antioxidant beneficial effects, as measured by increases in cytochrome oxidase and superoxide dismutase activities. Increases in cerebral blood flow and cognitive functions induced by LLLT have also been observed in humans. Importantly, LLLT given at energy densities that exert beneficial effects does not induce adverse effects. This highlights the value of LLLT as a novel paradigm to treat visual, neurological, and psychological conditions, and supports that neuronal energy metabolism could constitute a major target for neurotherapeutics of the eye and brain.
\end{abstract}

Keywords: photobiomodulation, cytochrome oxidase, neurotherapeutics, retinal disease, neurological disease, cognitive and mood disorders

\section{Introduction}

Low-level light therapy (LLLT) has gained attention in recent years as a novel tool for experimental therapeutic applications in a variety of medical conditions. The current paradigm shift in the field of neurotherapeutics has allowed consideration of this innovative approach in attempts to modify the function of the nervous system. Patients, research laboratories, the media, and industry around the world are devoting attention to the potential therapeutic applications of LLLT in neurology and other medical fields that have traditionally had a limited therapeutic contribution to patient care. In recent years, the use of LLLT has extended beyond the realms of pain and wound healing,
Correspondence: F Gonzalez-Lima University of Texas at Austin, I University Station A8000, Austin, TX 787 I2, USA

Tel + I 5 I 2 47 I 5895

$\mathrm{Fax}+|5| 2$ 47| 5935

Email gonzalez-lima@mail.utexas.edu 
and recent research supports its potential benefits in retinal disease, stroke, neurodegeneration, neuromuscular disorders, and memory and mood disorders. This therapeutic revolution is being favored by progress in the field of photobiology, aided by a twenty-first century reemergence of interest in bioenergetics. Current progress in photochemistry, genetics, informatics, and neuroimaging has allowed quantifying and differentiating the effects of light and other forms of electromagnetic radiation on biological tissues at different levels of analysis.

Throughout its development in the last 40 years, the concept of using "light to heal" has had an esoteric and suspicious connotation to the western contemporary biomedical mind. As illustrated by a reviewer's comments from a reputable biomedical journal to a recent manuscript on LLLT, the "curious effects" of light therapy have had the misfortune of being classified as a laughable school of thought in the tradition of astrology and Mesmer's animal magnetism. We are constantly exposed to light with apparently innocuous or trivial biological effects. Even when biological effects of light can be demonstrated, these are highly variable, present nontraditional dose-response curves, or lack a mechanistic explanation within traditional pharmacodynamic paradigms. A recent review stated that "widespread uncertainty and confusion exists about the mechanisms of action of LLLT at the molecular, cellular, and tissue levels." Thus, it is not surprising that LLLT lacks scientific appeal and has been denied entrance into mainstream medicine. Even when the benefit of doubt is allowed, LLLT could easily be regarded as a science-fiction construct or wishful thinking. Yet, compelling data on the potential clinical value of LLLT is available. A sound theory on the mechanism of action of LLLT implicating regulation of mitochondrial function has been advanced, and available data support that light-tissue interactions have special implications in highly metabolically-active excitable tissues, including the retina and the brain. Although there is still a lot to learn about mechanistic light-tissue interactions in the nervous system and the retina, evidence shows that LLLT can enhance neural metabolism by regulating mitochondrial function, intraneuronal signaling systems, and redox states. This review will briefly describe the current proposed photochemical mechanisms underlying the neurobiological effects of LLLT. A summary of current knowledge about LLLT dosimetry relevant for its variable effects in the nervous system, including noninvasive in vivo transcranial effects is also presented. A summary of key in vitro, preclinical, and clinical studies supporting the protective and enhancing effects of LLLT in a number of pathogenic conditions including cytotoxicity, mitochondrial dysfunction, and hypoxia/ischemia in the retina and the central nervous system is presented. The data on LLLT suggest it can exert effective, reproducible, and meaningful changes in the normal and dysfunctional nervous tissue. This highlights the value of LLLT as a novel and useful paradigm to treat visual, neurological, and psychological conditions, and supports that neuronal energy metabolism could constitute a major target for neurotherapeutics of the eye and brain.

\section{What is LLLT?}

Light is a type of electromagnetic radiation with both wave-like and particle-like properties. Living organisms are immersed in a vast ocean of electromagnetic radiation, which consists of periodic oscillations in electromagnetic fields that travel space and are thus able to transfer energy. Hence, light is a form of energy called luminous energy. A wave of electromagnetic radiation has a unidirectional vector and can be characterized in terms of its wavelength ( $\lambda=$ the distance between successive peaks or troughs), frequency (the number of oscillations per second), and amplitude (the difference between trough and peak). A complex mixture of waves with different frequencies, amplitudes, and wavelengths are absorbed, scattered, and reflected by objects, including biological material. Light of only one wavelength is called monochromatic. In modern quantum physics, electromagnetic radiation consists of photons, which are particles (quanta) of energy that travel at a speed of $3 \times 10^{8} \mathrm{~m} / \mathrm{second}$. The brightness of light is the number of photons and the color of the light is the energy contained in each photon. LLLT can be defined as the use of directional low-power and high-fluence monochromatic or quasimonochromatic light from lasers or light-emitting diodes (LEDs) in the red to near-infrared wavelengths $(\lambda=600-1100 \mathrm{~nm})$ to modulate a biological function or induce a therapeutic effect in a nondestructive and nonthermal manner. ${ }^{2,3}$ The effects of LLLT implicate conversion of luminous energy to metabolic energy with a subsequent modulation of the biological functioning of cells. Thus, LLLT is commonly known as photobiomodulation. It could also be called photoneuromodulation when nerve cells are the target. LLLT differs from the conventional effects of high-energy photon delivery commonly associated with lasers, which are mediated by a greater release of energy and result in heating and tissue destruction through dissection, ablation, coagulation, and vaporization. Compared to 
these commonly known destructive effects of lasers, LLLT is catalogued as "low-level" because the energy content of electromagnetic radiation is inversely proportional to its wavelength. In addition, the target tissue is generally exposed to low irradiances (ie, low Watts per $\mathrm{cm}^{2}$ of tissue), when compared to the energy delivered for ablative applications. Energy doses delivered by LLLT are too low to cause concerns about heating and tissue destruction, yet they are high enough to modulate cell functions. In fact, the typical irradiances used for photobiomodulation applications overlap with those used in topical photodynamic therapy for skin conditions. ${ }^{4}$ Early experiments demonstrated that photoneuromodulation of electrical activity in neurons can be achieved independently of thermal effects. ${ }^{5}$

Although cells in vitro are responsive to a variety of wavelengths in the electromagnetic spectrum, beneficial responses in vivo are observed preferentially within a more narrow wavelength range. Obviously, visible light (400-700 nm) penetrates the eyes and activates retinal cells that contain specialized photopigments (rods, cones, and some ganglion cells). But it is unknown whether in vivo exposure to light below $600 \mathrm{~nm}$ (such as blue or green light) can have beneficial effects on other nerve cells that are not specialized for photoreception. The red to near-infrared wavelength range has shown to be the most effective at inducing in vivo beneficial effects in cells that do not appear to have specialized photopigments. This is attributed in part to the capacity of different wavelengths to penetrate tissue: lower wavelengths such as violet and ultraviolet appear to penetrate less, whereas those in the red and infrared range have higher penetration. Also, energy at wavelengths shorter than $600 \mathrm{~nm}$ is generally scattered in biological tissues in vivo and they tend to be absorbed by melanin, whereas water significantly absorbs energy at wavelengths higher than $1150 \mathrm{~nm} .{ }^{6}$ For clinical purposes, this implies the existence of an in vivo therapeutic "optical window" that corresponds to red and near-infrared wavelengths. As discussed below, this window also matches the ability of luminous energy to excite susceptible intracellular molecules. ${ }^{6}$ For this reason, LLLT has also been referred to as near-infrared light therapy. LLLT is based on the principle that certain molecules in living systems are able to absorb photons and trigger signaling pathways in response to light. ${ }^{7}$ This process is termed energy conversion, and implies that the molecule excited by light reaches an electronically excited state that temporarily changes its conformation and function. In turn, this induces activation of signaling pathways that affect cellular metabolism.

\section{Photobiology of LLLT Properties of LLLT}

The major source of electromagnetic radiation in the environment is sunlight. Solar energy contains a rich combination of waves within the electromagnetic spectrum, including all wavelengths in the visible spectrum. Solar energy is multidirectional and noncoherent, which means that energy waves are not synchronized in space and time. LLLT differs from solar energy in that it is monochromatic and allows for potential high specificity and targeted molecular biomodulation (Figure 1). On the other hand, lasers feature monochromatic, unidirectional, and coherent electromagnetic radiation, which allows delivery of significant levels of concentrated energy. Because of this, many biomedical applications of lasers are characterized by the destructive effects of energy over very discrete areas of tissues. The advantages of lasers include high tissue penetration, their efficient fiber optic coupling, and high monochromaticity.

LLLT can be produced by LED arrays as well as lasers. Both sources have been used for photobiomodulation of the eye and brain. Laser sources produce $100 \%$ of coherent light energy in a single wavelength. They allow high tissue
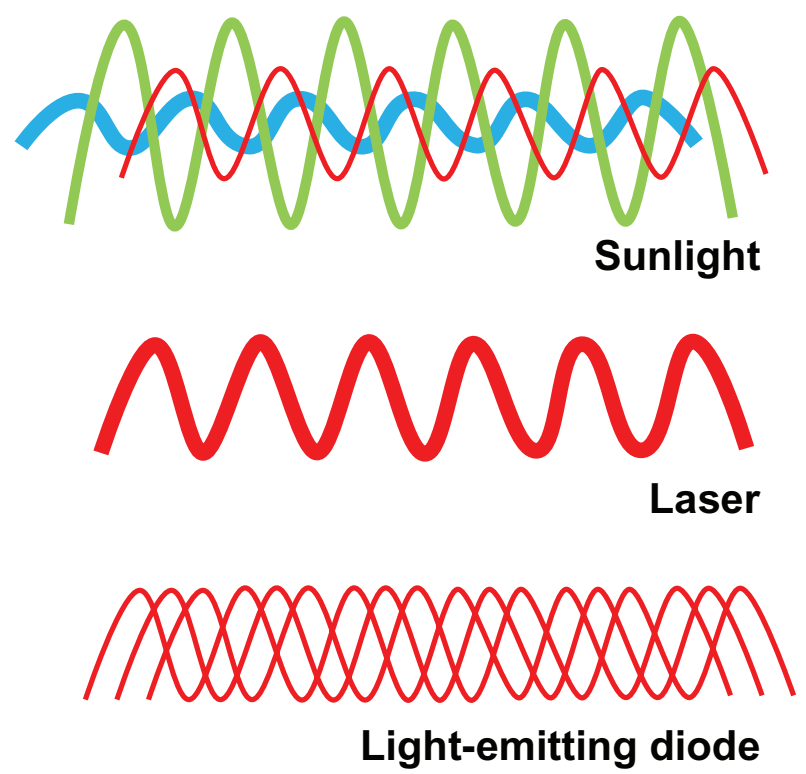

Figure I Properties of low-level light. Sunlight is composed of a combination of noncoherent waves with wavelengths spanning the entire visible spectrum. In contrast, lasers emit waves of a single wavelength (monochromatic) that have spatial and temporal synchronization. This high wavelength coherence allows the transmission of energy at a high power density. Finally, low-level light consists of monochromatic or quasimonochromatic waves taking different paths leading to a common target point. While wavelength, radiant exposure, irradiance, and fractionation scheme are relevant for low-level light therapy applications, the authors introduce the possibility that noncoherence may be advantageous for some neurometabolic purposes. Noncoherence allows nervous tissue exposure at "therapeutic" wavelengths at relatively low power densities during the time necessary to modulate neural metabolism in response to activation or injury, even if this time is prolonged. 
penetration and they produce a constant beam width that offers the advantage of energy delivery on circumscribed areas. For example, a Food and Drug Administration (FDA)cleared laser known as HD Laser $^{\mathrm{TM}}$ CG5000 (HD Laser Center, Dallas, TX) has a beam size of $45 \mathrm{~mm}$, a power density range up to $1.6 \mathrm{~W} / \mathrm{cm}^{2}$ and a $1064 \mathrm{~nm}$ wavelength that maximizes tissue penetration. The beam width of lasers can be modified by coupling them into fiber optic, which allows delivering energy to larger areas. Yet, areas of tissues that can be treated with lasers could be insufficient for some transcranial applications, and repeated single beam exposures are usually necessary. LEDs produce about 95\% of light between a narrow range of wavelengths (4-10 nm) and light is not coherent. Beam noncoherence of LEDs accounts for the significant difference in the amount of energy delivered to a single $\mathrm{cm}^{2}$ of target surface compared to lasers. While lasers are capable of heat production that can induce tissue damage, LEDs generate negligible amounts of heat, thus reducing the risk of thermal injury. ${ }^{8}$ But there is no risk of thermal injury at low irradiances if the laser output power is chosen correctly. LEDs can be mounted on arrays with ergonomic features that allow efficient energy delivery, which is relevant when the target organ has a large surface area, such as the brain. LED arrays and diode lasers are compact and portable, which is relevant in a clinical setting, and LEDs have achieved nonsignificant risk status for human trials by the FDA. ${ }^{9}$

Major dosimetric parameters relevant for LLLT studies are starting to become more standardized. Confusion about the effects of LLLT is evident in the literature, and it derives from lack of standardization of parameters relevant for LLLT. Until more is known about the dosimetry, the convention should be to report all relevant parameters involved in a particular LLLT use (Table 1, Figure 2). In contrast to traditional pharmacology, in which dose is a major determinant of the effect, LLLT is also dependent on power density, energy density, frequency, fractionation, wavelength, contact modality, source, and physicochemical properties of the target tissue.

\section{Hormetic effects of LLLT}

An accurate description of LLLT dosimetry should take into account the dose-response phenomenon of hormesis. A hormetic dose-response (also known as U-shaped,

Table I Major parameters of low-level light therapy (LLLT)

\begin{tabular}{|c|c|c|}
\hline Parameter & Unit & Explanation \\
\hline Wavelength & $\mathrm{nm}$ (nanometers) & $\begin{array}{l}\text { Wavelength }(\lambda) \text { is the distance between wave peaks. Light is a form of energy with } \\
\text { wave behavior. Photoacceptors exhibit different sensitivities to different wavelengths. } \\
\text { The most effective LLLT wavelength range is } 600-1100 \mathrm{~nm} \text {. Light visible to the human } \\
\text { eye is } 400-700 \mathrm{~nm} \text {. The higher the wavelength the lower the energy. }\end{array}$ \\
\hline Energy & J (joules) & $\begin{array}{l}\text { Energy }(E) \text { is the frequency }(v) \text { of radiation by Planck's constant }(h) \text { of } \\
6.626 \times 10^{-34} \mathrm{~J} \sec (E=h v) \text {. Energy of a photon depends on the frequency } \\
\text { of radiation (Ephoton }=h v) \text {. A photon is a particle of electromagnetic radiation } \\
\text { with zero mass and a quantum of energy (minimum } E \text { gained or lost by atom). } \\
\text { Energy }(J)=\text { Power }(\mathrm{W}) \times \text { Time (seconds). }\end{array}$ \\
\hline Power & W (Watts) & Amount of energy $(\mathrm{J})$ transferred or flowing per unit of time $(\mathrm{W}=\mathrm{J} / \mathrm{seconds})$. \\
\hline Irradiance & $\mathrm{W} / \mathrm{cm}^{2}$ & $\begin{array}{l}\text { Power }(\mathrm{W}) \text { per surface area }\left(\mathrm{cm}^{2}\right) \text {. Also called power density or light "intensity". } \\
\text { Irradiance = Power }(\mathrm{W}) / \text { Area }\left(\mathrm{cm}^{2}\right) \text {. }\end{array}$ \\
\hline Radiant exposure & $\mathrm{J} / \mathrm{cm}^{2}$ & $\begin{array}{l}\text { Energy }(\mathrm{J}) \text { per surface area }\left(\mathrm{cm}^{2}\right) \text {. Equivalent to power density per unit of time } \\
\text { (seconds). Also called fluence, energy density, or light "dose." Thus, "dose" can be } \\
\text { easily varied by changes in exposure time. However, at the same energy density } \\
\left(\mathrm{J} / \mathrm{cm}^{2}\right) \text { variations in either irradiance }\left(\mathrm{W} / \mathrm{cm}^{2}\right) \text { or time may cause different } \\
\text { LLLT effects on tissues. }\end{array}$ \\
\hline Exposure time & Seconds & Time during which the target tissue is exposed to light. \\
\hline Wave type & Continuous versus pulsed & $\begin{array}{l}\text { Continuous waves may be advantageous for transcranial applications. Pulse waves } \\
\text { may decrease thermal effects. Pulse Average Power }=\text { Peak Power }(\mathrm{W}) \times \text { Pulse Width } \\
(\text { seconds }) \times \text { Pulse Frequency }(\mathrm{Hz}) \text {. }\end{array}$ \\
\hline Fraction protocol & Number of fractions & $\begin{array}{l}\text { Total dose can be divided in treatment sessions or fractions of specific duration } \\
\text { and separated by specific intervals of time (eg, minutes, hours, days). }\end{array}$ \\
\hline Aperture & Area of the light beam & $\begin{array}{l}\text { Can be parallel, convergent, or divergent. Aperture may influence efficiency and } \\
\text { tissue penetration. }\end{array}$ \\
\hline Delivery mode & $\begin{array}{l}\text { Distance of the beam source } \\
\text { to the target tissue }\end{array}$ & $\begin{array}{l}\text { Types: shallow (or noncontact), contact, and deep. Shallow is preferable when larger } \\
\text { areas need to be exposed, but offers lower tissue penetration for light-emitting } \\
\text { diodes. Deep delivery implicates pressure of the beam source on the target tissue. }\end{array}$ \\
\hline
\end{tabular}



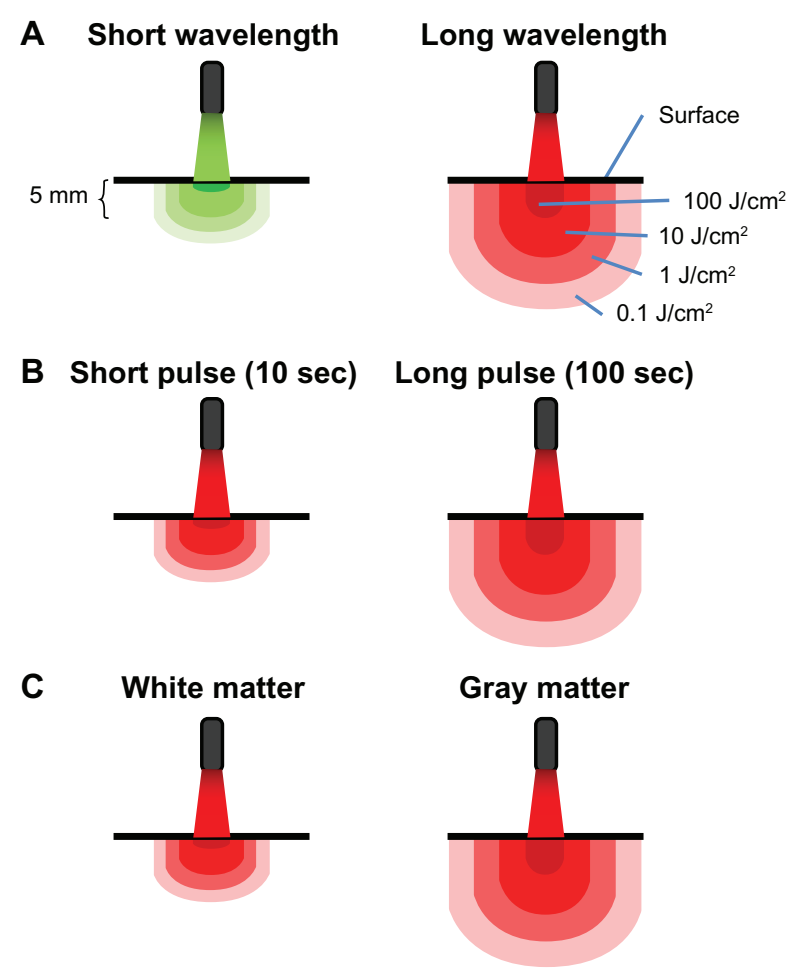

Figure 2 Principles of light-tissue interactions. (A) Light at short wavelengths has low tissue penetration. Light at high wavelengths displays high tissue penetration and delivers therapeutic levels of energy to deeper structures. Whereas surface structures exposed to high wavelengths may be exposed to inhibitory energy densities $\left(\mathrm{eg}, 100 \mathrm{~J} / \mathrm{cm}^{2}\right)$, light with a certain power density targeting a surface redistributes in a proportionally higher tissue volume due to diffraction (bending of waves). Multiple scattering of light allows for spreading out of waves and increases the treatment volume, so a lower applied energy can be used to achieve an effective energy density at higher depth. (B) Because radiant exposure $\left(\mathrm{J} / \mathrm{cm}^{2}\right)$ is the product of irradiance $\left(\mathrm{W} / \mathrm{cm}^{2}\right)$ and time, the energy delivered to tissues as a result of a constant irradiance can be increased by increasing exposure time. Thus, tissue penetration can also be affected by exposure time. When sources of low-level light therapy are used with high exposure times, deep structures can be treated with biomodulatory amounts of energy, while avoiding ablative effects. (C) Finally, tissues vary in their photoacceptor content, transmittance, and relaxation time. This accounts for interspecies and interregional variations in light penetration (eg, gray matter versus white matter in the brain). In addition, metabolically active tissues such as nervous tissue may exhibit variations in relaxation times, due to changes in the redox states of photoacceptors. This not only potentially affects tissue penetration, but also the susceptibility of nervous tissues to low-level light therapy depending on their activational state.

biphasic, or bell-shaped dose-response) is characterized by stimulation of a biological process at a low dose and inhibition of that process at a high dose (Figure 3). Hormetic models are superior to linear threshold models in their capacity to accurately predict responses below a pharmacological threshold. ${ }^{10}$ This is very relevant because the stimulatory responses to LLLT are usually modest, being only about $30 \%-60 \%$ greater than control values. This contrasts with the several-fold increases in a specific variable expected according to traditional dose-response models. The doseresponse phenomenon of hormesis is well documented in LLLT applications, since photostimulatory or photoinhibitory effects are obtained with low $\left(0.001-10 \mathrm{~J} / \mathrm{cm}^{2}\right)$ and high

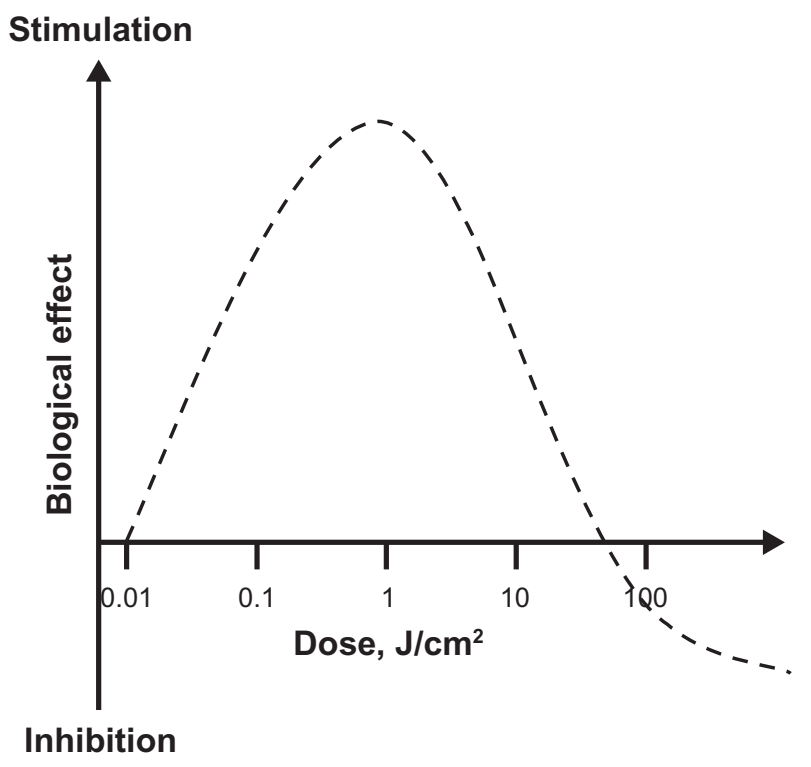

Figure 3 Hormetic effects of low-level light therapy (LLLT). LLLT does not induce classical linear dose-response pharmacological effects. LLLT effects are characterized by inverted $U$-shaped dose-response curves, in which linear responses may be seen only at very low doses. Whereas linear effects may be negligible, maximal stimulatory effects are typically observed at intermediate doses. However, the linear relationship does not hold at high doses, since inhibitory effects are observed instead. In fact, the inhibitory effects of very high LLLT doses might be worse than control conditions (eg, tissue destruction). A key observation concerning the modulatory effects of light in tissues is that maximal responses at intermediate doses tend to represent less than twofold increases in biological variables relative to baseline conditions. Yet these effects have been shown to have major relevance, especially when energy metabolism is involved in nervous tissue. Thus, hormesis is an essential concept for the development of neurotherapeutic applications of LLLT.

$\left(>10 \mathrm{~J} / \mathrm{cm}^{2}\right)$ energy densities, respectively. ${ }^{6}$ The achievement of positive responses is expected to vary within this dose range for a particular desired outcome. .11,12,9 $^{2}$

\section{Photoreceptors and photoacceptors}

Given the abundance of electromagnetic radiation, it is not surprising that organic systems have evolved mechanisms to use it and sustain biological functions. The uptake of electromagnetic radiation is, in fact, a critical process that makes life on Earth possible. Light energy uptake by living cells is based on the existence of biomolecules that can be excited by light quanta. Excitation of such molecules by electromagnetic radiation with the subsequent conversion of energy is the condicio sine qua non for any given photobiological effect. It has been shown that biological systems natively contain two types of molecules that can absorb light: specialized and nonspecialized. Highly efficient molecules with a significant degree of specialization for energy conversion are known as photoreceptors. These include photopigment molecules such as chlorophyll (important in the process of photosynthesis in plants), rod and cone opsins (retinal photoreceptors crucial for visual function), and melanopsin (found in some 
retinal ganglion cells that are important in the regulation of biological clocks).

On the other hand, nonspecialized molecules that can absorb light but are not integral to light receptor organs are called photoacceptors. Although photoacceptors can potentially absorb light, they usually are part of metabolic pathways not directly related with light processing. Photoacceptors are more ubiquitous and abundant than photoreceptors. In fact, photoacceptors can be artificially introduced into living systems, an early concept that led Niels Finsen to the development of phototherapy for which he was awarded a Nobel prize in Physiology or Medicine in 1903. ${ }^{13}$ Finsen's work has been the basis of photodynamic therapy and photochemotherapy (eg, photodynamic therapy and psoralen ultraviolet treatment), which are widely used in modern medicine mainly for the treatment of skin conditions. A central concept in the theory of LLLT photobiology is that its mechanism of action is mediated primarily by modulation of photoacceptor function. The identification and characterization of the properties of photoacceptors is a work in progress in the field of photobiology, but recent studies in photobiology support that effective stimulation of photoacceptors can induce effects of physiological and clinical relevance. This notion stems from the fact that photoacceptor molecules that are able to absorb light and exert changes in the function or structure of the cell are found in practically all living cells and not only in those of specialized sensory organs such as the eye (Figure 4).

Another important concept in the photobiology of LLLT is that of a chromophore. A chromophore is a particular moiety within a photoreceptor or photoacceptor molecule that is responsible for the absorption of light. Chromophores are usually organic cofactors or metal ions within a protein structure and contain electrons that can be excited from ground state to excited state, according to Boltzmann law. ${ }^{14}$ Excitation induces a molecular conformational change that is linked to changes in molecular function and intracellular metabolism. In cells, chromophores can consist of resonating systems or metal complexes. In resonating systems, electron excitation by light occurs within a structure that alternates single and double bonds, such as retinal, the chromophore linked to rhodopsin. Alternatively, in metal complexes, electron excitation occurs in open or closed pyrrole rings that allow binding of transition metals. The association of chromophores with respiratory enzymes has major implications for functional neural regulation. The unusual redox reactivity of chromophores is structurally coupled to protein distortion. In the case of chromophore-containing enzymes,

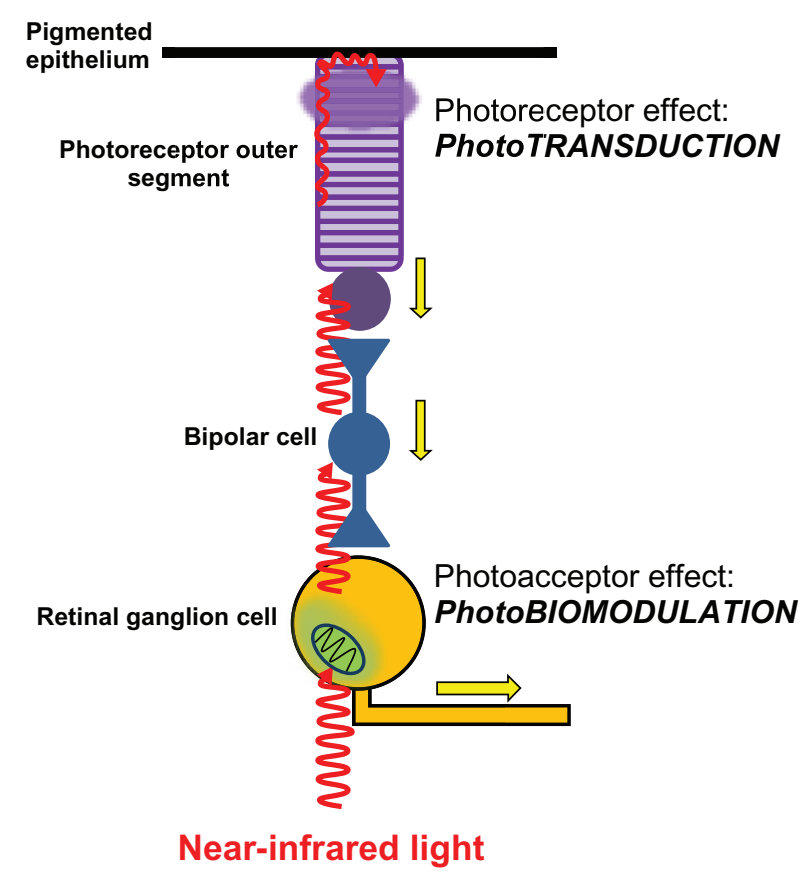

Figure 4 Differential effects of light on photoreceptors and photoacceptors in the retina. Light reaches the retina and travels through the different retinal layers to reach the outermost photoreceptor layer. It then excites the photoreceptor rhodopsin in rods and cones, triggering the process of phototransduction. Phototransduction causes photoreceptor cell hyperpolarization, changes in neurotransmission, and action potentials (yellow arrows) in bipolar cells and ganglion cells. These effects represent the onset of visual information processing. Light can also directly excite photoacceptors in neurons including retinal ganglion cells. The main photoacceptor in the red to near-infrared spectrum is the mitochondrial respiratory enzyme cytochrome oxidase. The effects of light on neuronal cytochrome oxidase induce modulation of cell bioenergetic mechanisms that are independent from visual processing. Yet photobiomodulation has major implications in neuronal physiology and homeostasis.

this allows a very efficient coupling of electromagnetic free energy flow to the chemical energy flow of substrate conversion. ${ }^{14,15}$ As discussed below, it is a remarkable finding that in mammalian tissues, the photosensitivity of certain chromophores conserves physiologic significance, in spite of their adaptation to naturally function without external photons. ${ }^{16}$ A distinctive property of chromophores is that they absorb particular wavelengths and reflect others, thus conferring specific colors to molecules. For example the porphyrin ring in chlorophyll a has peaks of absorption at $430 \mathrm{~nm}$ and $662 \mathrm{~nm}$. Similarly, the cones in the human retina contain one of three opsins with different spectral sensitivities and linked to the chromophore 11-cis-retinal or 11-cis-3,4dihydroretinal, which are derivatives of vitamin A. Thus, there are cones that are maximally sensitive to wavelengths of $430 \mathrm{~nm}$ (blue), $530 \mathrm{~nm}$ (green), and $560 \mathrm{~nm}$ (red). According to the Young-Helmholtz trichromatic theory, the color that we perceive is largely determined by the relative contributions of "blue, green, and red" signals sent from the cones in the retina to the brain. 
It is also well known that photoreception of light has important effects on biological systems independently of the engagement of visual function. Photoreception within the mammalian retina, including humans, is not restricted to the activity of rod and cone cells but extends to a small number of intrinsically photosensitive retinal ganglion cells. These ganglion cells in the inner layer of the retina serve as photoreceptors that provide information regarding environmental irradiance for a variety of nonimage-forming light responses including circadian entrainment and the pupillary reflex. ${ }^{17}$ These cells use another specialized photoreceptor molecule, melanopsin. Melanopsins absorb light through a retinaldehyde chromophore and drive changes in membrane potential via $G$ protein signaling cascades.$^{18}$ Interestingly, the pineal gland contains pinopsin, a photoreceptor similar to rhodopsin and melanopsin. It consists of an opsin molecule sensitive to blue light $(470 \mathrm{~nm})$ and a retinal chromophore. ${ }^{19}$ Pinopsin is exclusively expressed in the pineal gland and it is not expressed in the retina or other parts of the brain. It is believed that through pinopsin, environmental light resets the endogenous circadian pacemaker that controls the rhythmic production of melatonin independently of the visual pathway.

\section{Molecular biological targets of LLLT}

Early studies showed that LLLT has remarkable effects on living cells in cell cultures (in vitro effects). For example, LLLT-treated fibroblasts show accelerated metabolism, DNA synthesis, and growth rate. ${ }^{20}$ These notable positive effects on connective tissue in vitro led to the application of LLLT in the treatment of inflammation and improvement of wound healing in vivo. Since the notable effects of LLLT were first described, a question that has attracted a great deal of attention is what molecule or molecules in cells have the features to make them responsible for the effects of LLLT. As mentioned above, wavelengths in the red to near-infrared range have been found to produce the most significant responses in vivo and are consequently the best characterized in terms of their photobiological response. One of the better characterized photoacceptors in mammalian tissues is hemoglobin. Hemoglobin shows differential light absorption based on its redox state. This property allows its quantification and is the principle of pulse oxymetry and other methods of oxygenation monitoring in clinical practice. However, LLLT has shown beneficial effects in fibroblasts, epithelial cells, HeLa cells, and neurons in culture. Since hemoglobin is exclusively expressed in red blood cells, this suggests that alternate intracellular molecules different from hemoglobin should be the primary photoacceptors mediating LLLT effects. Besides hemoglobin, the most common photoacceptors in the red to near-infrared range are also hemecontaining metalloproteins: myoglobin and cytochrome oxidase. Nevertheless, other molecules such as superoxide dismutase, cytochrome c, cytochrome b, nitric oxide synthase, catalase, guanylate cyclase, and the cryptochromes have also been shown to have photoacceptor capacities. ${ }^{8}$ Elucidation of the mechanism of action of LLLT seems puzzling in view of the existence of different molecules with photoacceptor capabilities. This is complicated by the fact that the correspondence of a single molecular target to a particular effective wavelength is not straightforward. For example, flavoproteins such as the reduced nicotinamide adenine dinucleotide (NADH)-dehydrogenase have been identified as photoacceptors in both the violet-to-blue and red to near-infrared spectral region. ${ }^{21}$ At the same time, terminal oxidases and the endogenous antioxidant enzyme superoxide dismutase also show absorption peaks at high wavelengths $(670-680 \mathrm{~nm})$ which represents a clear overlap in the absorption spectrum of different photoacceptors..$^{21,22}$ This signifies that even when a particular molecule is found to mediate a biological effect for the most part, other possible photoacceptors can also contribute at least in some degree to elicit a particular response.

Notably, experiments on the nervous system were the first to provide a clue on the identity of the major photoacceptor mediating the effects of LLLT. Kato et $\mathrm{a}^{23}$ suggested that mitochondria in the bird brain could work as photoreceptors for a photobiological process relating to gonadal growth. Subsequent experiments demonstrated that isolated mitochondria are sensitive to irradiation with monochromatic light in the red and near-infrared spectrum. For example, illumination of isolated rat liver mitochondria increased adenosine triphosphate (ATP) synthesis and oxygen consumption. ${ }^{24}$ Light also increased the mitochondrial membrane potential $(\Delta \Psi)$ and proton gradient $(\Delta \mathrm{pH})$. Light alters mitochondrial optical properties, increases adenosine diphosphate/ATP exchange, ribonucleic acid (RNA) and protein synthesis in mitochondria, and increases oxygen consumption..$^{25}$ These effects of LLLT on mitochondria are observed in a wavelength-specific manner. These data suggested not only that the primary photoacceptor mediating the effects of LLLT is localized to mitochondria, but that molecules that absorb LLLT in cells are probably components of the respiratory chain. ${ }^{25}$

Further identification of mitochondrial photoacceptors was done by means of action spectra analysis. An action spectrum is the description of a biological response to LLLT 
as a function of wavelength. For example, the rate of RNA synthesis in HeLa cells in culture can be increased at certain wavelengths, whereas other wavelengths exert no effect. Usually, a range of wavelengths (ie, band) is effective at inducing the response, and the wavelength that induces a maximal effect can be found within this range. The bands in the action spectra can then be compared with those of the metal-ligand system absorption spectra of visible to near-infrared spectral range. This method of action spectra analysis is based on analogy and does not distinguish between photoacceptors with very similar absorption spectra. However, its use provided major hints for identifying the main photoacceptor mediating the effects of LLLT. The action spectra of RNA synthesis in HeLa cells shows several peaks ranging from $400-820 \mathrm{~nm}$. The range $580-860 \mathrm{~nm}$ shows two doublet bands in the ranges $620-680 \mathrm{~nm}$ and $760-830 \mathrm{~nm}$ with well-defined maxima at $620 \mathrm{~nm}, 680 \mathrm{~nm}, 770 \mathrm{~nm}$, and $820 \mathrm{~nm}$. Comparative analysis of spectral data for transition metals and their complexes with biomolecules participating in regulation of cellular metabolism revealed that regions $400-450 \mathrm{~nm}$ and $620-680 \mathrm{~nm}$ of the spectra match those of complexes with charge transfer in a metal ligand system. The data suggested two possible scenarios: either multiple photoacceptors are responsible for this spectral pattern, or a single photoacceptor molecule presents multiple metal-ligand chromophores with absorption peaks at 420-450 nm and 760-830 nm. Consistent with the second option, all bands in the action spectrum match the absorption spectrum of the mitochondrial enzyme cytochrome oxidase. ${ }^{21}$

It is currently accepted that cytochrome oxidase is the primary photoacceptor of light in the red to near-infrared region of the electromagnetic spectrum. ${ }^{26,27}$ Cytochrome oxidase is a key enzyme for cell bioenergetics, especially for nerve cells in the retina and brain. ${ }^{28}$ It is the terminal complex of the mitochondrial electron transport chain and catalyzes electron transfer from cytochrome c to molecular oxygen, accomplishing reduction of more than $95 \%$ of the oxygen taken up by eukaryotic cells. Cytochrome oxidase constitutes an efficient energy-transducing device, acting as both a redox-linked proton pump that creates a transmembrane electrochemical gradient, and a rate-limiting step for the synthesis of the energy-storing molecule ATP. ${ }^{29}$ Cytochrome oxidase is a large multicomponent membrane protein of considerable structural complexity (molecular size $200 \mathrm{kDa}$ ). It is a bigenomicallyregulated enzyme and its expression is tightly coupled to neuronal energy demands, free radical metabolism, cell death pathways, and glutamatergic activation. ${ }^{30,31}$ Its activity has been extensively used to reliably quantify neuronal function and it represents the best known intraneuronal marker of metabolic activity. ${ }^{31}$ The photochemistry of cytochrome oxidase as the primary photoacceptor of LLLT has been extensively characterized. Cytochrome oxidase contains four redox metal centers: $\mathrm{Cu}_{\mathrm{A}}, \mathrm{Cu}_{\mathrm{B}}, \mathrm{Hem} \mathrm{a}$, and Hem $\mathrm{a}_{3}$. In the catalytic cycle of cytochrome oxidase, electrons are transferred sequentially from water-soluble cytochrome $\mathrm{c}$ to $\mathrm{Cu}_{\mathrm{A}}$, then to Hem a and to the binuclear center $\mathrm{a}_{3}-\mathrm{Cu}_{\mathrm{B}}$ where oxygen is reduced to water. These metal centers determine different light absorption peaks for the enzyme: $620 \mathrm{~nm}$ (range 613.5-623.5 nm), $825 \mathrm{~nm}$ (range 812.5-846 nm), $760 \mathrm{~nm}$ (range 750.7-772.3 nm), and $680 \mathrm{~nm}$ (range 667.5-683.7 nm), which correspond to $\mathrm{Cu}_{\mathrm{A}}$ reduced, $\mathrm{Cu}_{\mathrm{A}}$ oxidized, $\mathrm{Cu}_{\mathrm{B}}$ reduced, and $\mathrm{Cu}_{\mathrm{B}}$ oxidized, respectively. ${ }^{24}$ The redox state of the enzyme can vary from fully reduced to fully oxidized, with intermediate states that include oxidation of one, two, or three metal centers (mixed valence enzyme). Electronic excitation of these centers in a particular sequence has a differential influence on the electron flow within cytochrome oxidase. This has a direct photobiological correlate as demonstrated by changes in the rate of DNA synthesis. Results of experiments using sequential irradiation have shown that cytochrome oxidase cannot be a primary photoacceptor when it is fully reduced or fully oxidized, but only when it is in one of its intermediate forms. ${ }^{32,33}$ Whereas the fully oxidized or reduced forms are insensitive to LLLT, the partially reduced enzyme showed an increase in absorbance and its proton pump activity upon LLLT. ${ }^{26}$ In addition, simultaneous dichromatic irradiation changes the ratio of the reduced and oxidized forms of the enzyme. Thus, it is suboptimal at inducing a biological response, compared to the use of single wavelengths in the red to near-infrared range. The absorption spectra of cytochrome oxidase in different oxidation states have been found to parallel the action spectra (photoresponse as a function of wavelength) of biological responses to LLLT. ${ }^{32}$ In neural tissue, cytochrome oxidase is the most abundant metalloprotein and wavelength peaks in its absorption spectrum $(670 \mathrm{~nm}$ and $830 \mathrm{~nm})$ highly correlate with its peaks in catalytic activity and with ATP content in vitro. ${ }^{8}$

\section{Mechanism of action of LLLT}

It is easy to reconcile the fact that cytochrome oxidase is the primary photoacceptor of LLLT with beneficial eye and brain effects since this enzyme is a key molecule involved in oxidative energy metabolism, and neurons depend on cytochrome oxidase to produce their metabolic energy. Modulation of this major component of the cell respiration system can be anticipated to exert profound effects in whole-cell 
physiology. Consistent with this idea, a number of intracellular effects have been described during and after light excitation. Based on these data, a mechanistic hypothesis on the mechanism of action of LLLT has been advanced. For study purposes, the mechanistic effects of LLLT can be divided into primary (during light exposure) and secondary (after light exposure).

\section{Primary effects}

Primary effects refer to the direct photochemical change occurring in the photoacceptor upon excitation by light. Primary effects are light-dependent and they occur only while the target tissue is being exposed to light. Current evidence is available to support at least three different primary effects. The first and most important primary effect is a redox change of the components of the respiratory chain. LLLT can induce reduction or oxidation of cytochrome oxidase. These changes in redox status correlate with the bell-shaped doseresponse recorded for cellular responses. ${ }^{3}$ Changes in the redox status of cytochrome oxidase implicate alterations in electron flow. LLLT increases cytochrome c oxidation in the presence of cytochrome oxidase, causes increases in oxygen consumption and mitochondrial membrane potential, and activates the mitochondrial permeability transition pore. ${ }^{21,20}$ All of these events have been associated with accelerated electron flow in the mitochondrial electron transport chain. The second possible primary effect is the generation of free radicals, including singlet oxygen via direct photodynamic action and superoxide ion via one electron auto-oxidation. The significance of this effect is that reactive oxygen species are not only damaging by-products of respiration but they have an important role in cellular signaling. The third primary effect of LLLT is localized transient "heating" of the absorbing chromophore based on electric or light oscillations..$^{21}$ This effect has been less characterized and it is believed to complement the other two proposed primary effects. The effect of such oscillations appears to be more generalized and affect all molecules in the target tissue, including water. LLLT is able to strengthen hydrogen bonds and induce large-size hydrogen bonds networks that allow quick energy transfers due to resonant intermolecular energy transference. Thus, LLLT can cause nonequilibrium electrical fluctuations that bias Brownian motion and induce mechanisms that support electron pumping without heat transfer. ${ }^{14}$

\section{Secondary effects}

The secondary effects of LLLT occur as a consequence of primary effects and include a cascade of biochemical reactions that change cellular homeostasis. ${ }^{34,35}$ Secondary effects feature activation of second messengers with subsequent modulation of enzyme function and gene expression. Secondary effects are distinctive because they can occur hours and even days after light exposure and they implicate the activation of signaling pathways that result in amplified macroeffects. Because they occur as part of cascade reactions, secondary effects tend to be pleiotropic. LLLT activates the retrograde signaling pathway from the mitochondria to the nucleus. This signaling pathway induces adaptive responses to stress by sending information from mitochondria, which contain the photoacceptors, to the nucleus, which can then respond by altering levels of gene expression. The initial phase of this pathway has been proposed to be an increase in the NAD/NADH ratio and mitochondrial intermembrane potential, dissociation of nitric oxide from its binding site in cytochrome oxidase, and modification of the ATP pool. Even small changes in ATP alter cellular metabolism. ATP activates $\mathrm{P} 2$ receptors ( $\mathrm{P} 2 \mathrm{X}$ and $\mathrm{P} 2 \mathrm{Y}$ ) to induce inward calcium currents and release of calcium from intracellular stores. ${ }^{35}$ Changes in ATP also alter cyclic adenosine monophosphate levels, with subsequent activation of kinases. Downstream consequences of these secondary effects involve changes in gene expression, which impact mitogenic signaling, surface molecule expression, and expression of proteins regulating inflammatory, redox states, and apoptosis (Figure 5).

\section{Effects of LLLT on nervous tissue}

A growing body of evidence supports an enhancing or protective role of LLLT in nervous tissue. Neurons are highly specialized cells with a major dependence on sustained aerobic energy production. Mitochondrial aerobic metabolism in neurons is the basis for electrophysiological, neuroplastic, and neuroprotective functions including repolarization of cell membranes in neurotransmission, synapse formation, and cell survival. At a large scale, energy metabolism is also crucial for adequate function of neuronal networks, data integration in space and time, and sensory processing including vision, activation of motor function, and expression of higher-order cognitive functions such as memory. It has been demonstrated that impaired mitochondrial oxidative metabolism is associated with neuronal dysfunction, neurological impairment, and neurodegeneration. ${ }^{36}$ Therefore, interventions aimed at improving mitochondrial metabolism are hypothesized to benefit the function of both the diseased and normal brain.

Recent studies have confirmed that the effects of LLLT on nervous tissue are also mediated at least in part by 


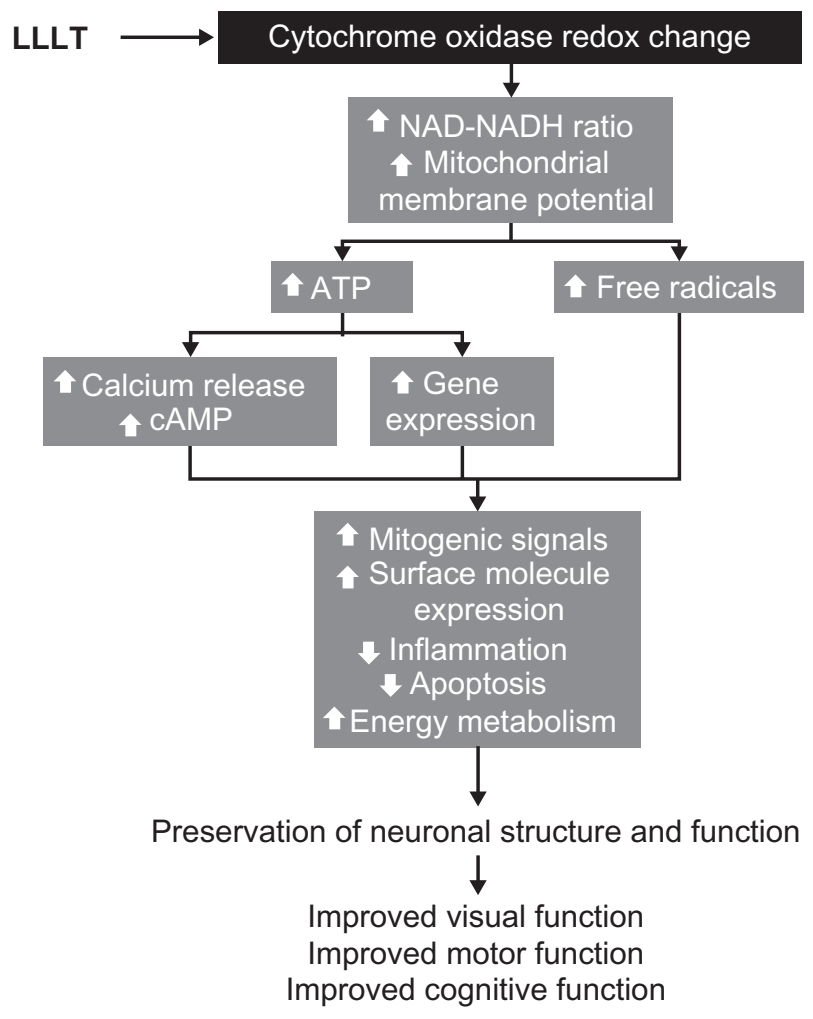

Figure $\mathbf{5}$ The intracellular mechanisms of action of low-level light therapy (LLLT). Photobiomodulation results in a cascade of intracellular pleiotropic effects. Light is absorbed by chromophores in cytochrome oxidase and induces changes in its redox state. Redox reaction of enzymes in the inner mitochondrial membrane induces accelerated electron flow, reduced nicotine adenine dinucleotide (NADH) consumption and increase in the mitochondrial membrane potential. These changes facilitate the synthesis of adenosine triphosphate (ATP) and increase the generation of free radicals. Increased ATP availability allows the activation of kinases that induce the release of calcium and the formation of cyclic adenosine monophosphate (cAMP). Calcium, cAMP, and free radicals act as second messengers and are able to activate different metabolic pathways at the nuclear level. Depending on the cell environment, these cellular changes can be adaptive and promote enhancement of neuronal physiology that translates in clinical improvement.

enhancement of cytochrome oxidase activity. Rat neuronal cultures exposed to LLLT showed increases in cytochrome oxidase activity. ${ }^{9}$ These increases of cytochrome oxidase induced by LLLT parallel those observed in other tissues with high metabolic demands such as muscle. ${ }^{37} \mathrm{~A}$ similar enhancing effect of LLLT was also observed when delivered after exposure of neurons in culture to tetradotoxin, which blocks electrical neural activity and indirectly inhibits cytochrome oxidase activity. ${ }^{38}$ In addition, LLLT partially restored enzyme activity blocked by potassium cyanide, a cytochrome oxidase inhibitor, and significantly reduced neuronal cell death. ${ }^{9}$ This illustrates that the enhancing effects of LLLT on neuronal metabolism are not limited to enhancement of cytochrome oxidase activity, but have the potential to exert extended effects such as enhancement of ATP production, ${ }^{36}$ neurotransmission, ${ }^{8}$ gene expression, ${ }^{38,39}$ and prevention of cell death in vitro. ${ }^{40,41}$
Protective effects were observed when striatal and cortical rat neuronal cultures were exposed to rotenone and 1-methyl4-phenylpyridinium $\left(\mathrm{MPP}^{+}\right)$toxins that inhibit mitochondrial complex I. LLLT significantly increased cellular ATP content, decreased the number of neurons undergoing cell death, and reduced the expressions of reactive oxygen species and reactive nitrogen species in rotenone- or $\mathrm{MPP}^{+}$-exposed neurons as compared with untreated ones. ${ }^{41}$ Furthermore prophylactic LLLT in vitro has proved very effective at protecting neurons from neurodegeneration induced by mitochondrial toxins..$^{9,36,38,40}$ Notably, LLLT pretreatment suppresses rotenone- or $\mathrm{MPP}^{+}$-induced apoptosis in both striatal and cortical neurons. ${ }^{38}$ LLLT has been also successfully employed for nerve repair and reduction of neural injury in animal models ${ }^{42}$ and it is clinically used to relieve pain in humans. ${ }^{43}$

A number of secondary effects of LLLT on nervous tissue have also been described, including: (1) increased expression of the anti-apoptotic protein $\mathrm{Bcl}-2$ and reduced expression of the proapoptotic protein Bax, ${ }^{38}$ (2) decreased numbers of apoptotic cells after exposure to the amyloid beta protein, ${ }^{44}$ (3) improved function of cortical neurons inactivated by toxins, ${ }^{9,38}$ (4) increased survival and ATP content of striatal neurons after rotenone- and $\mathrm{MPP}^{+}$-induced toxicity and decreased oxidative stress and nitric oxide production, ${ }^{40}$ (5) increased neurite outgrowth, ${ }^{45}$ (6) regulation of genes encoding for DNA repair proteins, antioxidant enzymes, and molecular chaperones, ${ }^{8}$ and (7) increased proliferation of olfactory ensheating stem cells, ${ }^{39}$ Schwann cells,${ }^{46}$ astrocytes, and oligodendrocytes. ${ }^{47}$ In vivo, LLLT induces peripheral and central nerve regeneration after trauma, ${ }^{42,47,48}$ reduces neuroinflammation, ${ }^{42}$ prevents methanol-induced photoreceptor degeneration, ${ }^{12}$ and prevents retinal neurodegeneration induced by complex I inhibition. ${ }^{49}$

\section{Beneficial in vivo effects of LLLT in the eye}

Light tissue penetration depends on both the type of target tissue, wavelength, and the source of LLLT (Figure 2). Besides being safe, at near-infrared wavelengths, light penetration to the eye is maximal, where absorption by the cornea and lens is negligible $(<10 \%)$ and high refractive indices favor low light scattering and a high degree of focusing on the retina. ${ }^{50}$ The retina contains neurons with extremely high energy demands that rely mostly on mitochondrial-derived ATP to meet these requirements. ${ }^{51-53}$ Mitochondria play a central role in neuronal physiology. These organelles integrate cell respiration, energy metabolism, and ionic balance into a 
homeostatically coherent adaptation for energy maintenance and cell survival. Thus, similar to other neuronal populations, retinal neurons are highly vulnerable to events that lead to oxidative stress and energy depletion, including oxygen or glucose deprivation and dysfunction of the mitochondrial machinery that uses both of them to generate ATP. ${ }^{54,55}$ Evidence accumulated in the last 30 years suggests that mitochondrial dysfunction, induced by both genetic and environmental factors, plays a key role in the pathogenesis of retinal neurodegeneration, the main morphological feature of optic neuropathy. ${ }^{56}$ Optic neuropathy is featured in conditions with high morbidity and mortality for which no effective treatments are available. ${ }^{57-60}$

Common eye disorders such as glaucoma and age-related macular degeneration, and even neurodegenerative disorders such as Alzheimer's disease, have been increasingly recognized to feature optic neuropathy induced by mitochondrial dysfunction. Patients with Alzheimer's disease show a reduction in the number of retinal ganglion cells and axons, compared to healthy individuals. ${ }^{60-62}$ In addition, the most common primary mitochondrial disorder, Leber's optic neuropathy, is responsible for approximately $2 \%$ of all cases of blindness. ${ }^{63}$ All these conditions feature retinal ganglion cell degeneration, optic nerve atrophy, and blindness that severely decrease the quality of life of affected individuals, and represent a major public health problem. A number of genetic and acquired conditions featuring blindness secondary to degeneration of the retina and optic nerve have also been linked to mitochondrial dysfunction. Besides Leber's hereditary optic neuropathy, genetic conditions featuring optic neuropathy and mitochondrial failure include Leigh syndrome, ${ }^{64,65}$ Friedreich's ataxia, ${ }^{59,66}$ myoclonic epilepsy ragged-red-fibers, ${ }^{67}$ mitochondrial encephalomyopathylactic acidosis and stroke-like syndrome, ${ }^{68}$ hereditary spastic paraplegia, ${ }^{69}$ and the deafness-dystonia-optic atrophy syndrome. ${ }^{70}$ Similarly, acquired diseases featuring optic neuropathy with an association with mitochondrial dysfunction include the tobacco-alcohol amblyopia, and intoxication with chloramphenicol, ethambutol, carbon monoxide, clioquinol, cyanide, hexachlorophene, isoniazid, lead, methanol, plasmocid, or triethyl tin. ${ }^{66}$ Thus, LLLT has potential significant applications as therapy against retinal damage by counteracting the immediate consequences of mitochondrial failure.

The beneficial effects of LLLT in the eye were tested in early studies using low energy helium-neon laser irradiation in models of traumatic optic nerve injury in the rabbit and rat. ${ }^{71,72}$ The function of the injured optic nerve, as measured by compound action potentials, showed a significant improvement after two weeks of daily radiation. Optic nerve function showed more significant improvements when LLLT was started immediately after injury, and efficacy was progressively lost if onset of treatment was delayed to 2 hours, 5 hours, and 24 hours. More significantly, LLLT started immediately before injury was also effective at delaying posttraumatic nerve damage. These studies were also significant because they demonstrated that LLLT was effective on moderately injured nerves, whereas its beneficial effects were not observed in severely-injured nerves. This suggests that a physiologically viable neural substrate capable of responding to light is needed. It is less likely that LLLT worked by inducing neuronal repair; rather, light may have worked by enhancing the function of spared nerve fibers. ${ }^{72}$

The first evidence that LLLT may offer retinal protection in situations of injury in which disruption of mitochondrial energy metabolism is a major mediator was demonstrated using a model of retinal injury induced by methanol. ${ }^{12}$ By being metabolized into formic acid, an inhibitor of cytochrome oxidase, methanol induced $72 \%$ decrease in retinal sensitivity to light and attenuation of the maximal electroretinogram response amplitude. These changes are consistent with photoreceptor toxicity. LLLT prevented the functional deficits induced by methanol, as shown by a decrease of only $28 \%$ in the electroretinogram responses. LLLT also protected the methanol-induced disruption of the retinal architecture. Methanol induced retinal edema, swelling of photoreceptor inner segments, including mitochondria, and morphologic changes in photoreceptor nuclei. In contrast, LLLT-cotreated rats showed retinal histology indistinguishable from control rats. These effects were induced by using LEDs at $670 \mathrm{~nm}$ and an energy density of $12 \mathrm{~J} / \mathrm{cm}^{2}$ divided into three fractions given 5 minutes, 25 minutes, and 50 hours after systemic methanol administration. These parameters are also known to be effective at inducing cell proliferation of visual neurons in culture and wound healing. ${ }^{12}$

A recent study showed evidence that LLLT could be effective at preventing the effects of phototoxicity, which has clinical relevance in the prevention of age-related macular degeneration. The photoreceptor protective effects of LLLT are also evident when damage is induced by phototoxicity. White light at $1800 \mathrm{~lx}$ for 3 hours causes significant damage to the outer nuclear layer of the retina in pigmented rats. This structural damage is accompanied by an attenuation of the $b$ wave in the electroretinogram. LLLT reduced the extension of damage in the outer nuclear layer and maintained the electroretinogram $b$ wave amplitude. ${ }^{73}$ LLLT has also been shown to prevent inflammation and photoreceptor damage 
induced by phototoxicity. LLLT at $670 \mathrm{~nm}, 9 \mathrm{~J} / \mathrm{cm}^{2}$, and $60 \mathrm{~mW} / \mathrm{cm}^{2}$ was given for 5 days before and 5 days after phototoxicity with bright light in albino rats. Retinal phototoxic damage features a $25 \%-75 \%$ thinning of the outer nuclear layer, degeneration of photoreceptors, loss of the retinal pigment epithelium, and microglial invasion. In this series of experiments, LLLT given before the phototoxic damage was highly effective at preventing the morphological alterations observed in the nontreated group. This study also showed evidence that the retinoprotective effects of LLLT against phototoxicity include reduction of microglial invasion, decrease of stress-related molecules, and increase in neuroprotective molecules. The protective effects of LLLT were also observed at the functional level as determined with flash-evoked electroretinography. Remarkably, the protective effects of LLLT were long-lasting and photoreceptor function was preserved as late as 1 month after damage. Also, LLLT alone did not have a significant effect on photoreceptor function and it did not affect the expression of stress-related factors. ${ }^{74}$ However, LLLT has been shown to be associated with an increase expression of RNA involved in neuroprotective roles. LLLT prevention of photoreceptor cell death induced by phototoxicity have been associated with a decrease in the expression of 126 of 175 genes upregulated by light damage. ${ }^{75}$

LLLT has also shown protective effects in a rat model of retinitis pigmentosa featuring a rhodopsin mutation inducing photoreceptor degeneration during development. Rat pups were treated with LLLT at $670 \mathrm{~nm}, 50 \mathrm{~mW} / \mathrm{cm}^{2}$, and $4 \mathrm{~J} / \mathrm{cm}^{2}$ for 5 days during the critical period of photoreceptor development. LLLT increased the concentrations of retinal cytochrome oxidase and the neuroprotective factors superoxide dismutase and ciliary neurotrophic factor. In this model, LLLT also decreased the rate of photoreceptor cell death by $70 \%{ }^{76}$

Recent evidence also supports that LLLT protects against retinal ganglion cell degeneration and that this effect is behaviorally meaningful. This has been demonstrated in an in vivo model of retinal degeneration induced by the mitochondrial complex I inhibitor rotenone..$^{49}$ Intravitreal administration of rotenone induces impairment of visual function that can be measured as a decrease in the illuminance sensitivity threshold. Rotenone also impairs the escape latency and the rate of successful trials in a two-choice visual task in rats. These behavioral impairments correlate with ganglion cell and retinal nerve fiber layer degeneration. These changes induce a functional deafferentiation that is evident as a decrease in the metabolic capacity of the retina and central visual pathway. All these functional and structural changes were prevented by LLLT $630 \mathrm{~nm}$ in a dose-dependent manner. A total high dose of $21.6 \mathrm{~J} / \mathrm{cm}^{2}$ given in six fractions (3.6 J/ $\mathrm{cm}^{2}$ per fraction, one fraction per day) after rotenone injection was effective at preventing visual dysfunction and retinal neurodegeneration. LLLT also prevented the metabolic deafferentiation caused by the neurotoxin, which was observed as preservation of the metabolic capacity in the superior colliculus, lateral geniculate nucleus, primary visual cortex, and secondary visual cortex. In contrast, a total low LLLT dose of $10.8 \mathrm{~J} / \mathrm{cm}^{2}$ given in three fractions $\left(3.6 \mathrm{~J} / \mathrm{cm}^{2}\right.$ per fraction) after rotenone injection was not as effective at preventing retinal degeneration. However, when retinas were treated prophylactically with LLLT 2 days prior to rotenone administration, the low LLLT dose was effective at preventing retinal neurodegeneration and visual dysfunction.

Primate studies have shown that LLLT is effective at decreasing the functional and morphological retinal injury induced by photocoagulation, when given 1 hour, 24 hours, 72 hours, and 96 hours after injury. ${ }^{76}$ In addition, an interventional case report describes a patient with bilateral atrophic age-related macular degeneration that showed improvement in visual acuity and appearance of previously undetected oscillatory potentials in electroretinogram recordings after LLLT. LLLT also induced a mobilization of drusen (yellow deposits in age-related macular degeneration) and a decrease in intraocular pressure, and these beneficial effects were evident several months after treatment. ${ }^{77}$ This evidence suggests that LLLT may induce beneficial effects in retinal and optic nerve pathology in humans (Table 2).

\section{Beneficial in vivo effects of LLLT in the brain \\ Transcranial effects}

The ability of light to penetrate the brain and exert biological effects was first inferred after finding photoreceptor molecules present in central nervous system structures. For example, the pineal gland contains the photoreceptor pinopsin and it is believed to be sensitive to ambient light changes and be relevant for the regulation of circadian rhythms. ${ }^{19}$ It has been demonstrated that light is able to penetrate the cranium and reach the brain. ${ }^{78}$ This effect is being used for the development of optical imaging techniques using nearinfrared light in humans. ${ }^{79}$ Although light attenuation occurs when light travels through bone, this attenuation is not of a strong magnitude. The cranium shows minimal absorption and scattering of light, with a wavelength- but not thicknessdependent attenuation of $15 \%-20 \%$ in the red to near-infrared 
Table 2 Beneficial in vivo effects of low-level light therapy on the eye

\begin{tabular}{|c|c|c|c|c|c|}
\hline Light source & Wavelength & Dose & Effect & Relevance & Reference \\
\hline He-Ne laser & $632.8 \mathrm{~nm}$ & $\begin{array}{l}10.5 \mathrm{~mW}, \mathrm{I} .1 \mathrm{~mm} \text { beam } \\
\text { diameter } \times 2 \text { minutes, } \\
\text { daily for } 2 \text { weeks }\end{array}$ & $\begin{array}{l}\text { Preserved structure and function } \\
\text { after optic nerve crushed injury } \\
\text { (rat, rabbit) }\end{array}$ & $\begin{array}{l}\text { Optic nerve } \\
\text { trauma }\end{array}$ & $\begin{array}{l}\text { Schwartz et al, }{ }^{71} \\
\text { Assia et al, }{ }^{72}\end{array}$ \\
\hline GaAIAs LED & $670 \mathrm{~nm}$ & $\begin{array}{l}28 \mathrm{~mW} / \mathrm{cm}^{2}, 12 \mathrm{~J} / \mathrm{cm}^{2} \\
\text { in three fractions }\end{array}$ & $\begin{array}{l}\text { Preserved structure and function } \\
\text { after systemic methanol } \\
\text { photoreceptor toxicity (rat) }\end{array}$ & $\begin{array}{l}\text { Methanol } \\
\text { intoxication }\end{array}$ & Eells et al, ${ }^{12}$ \\
\hline GaAlAs LED & $633 \mathrm{~nm}$ & $\begin{array}{l}2 \mathrm{~mW} / \mathrm{cm}^{2}, 21.6 \mathrm{~J} / \mathrm{cm}^{2} \\
\text { in six fractions }\end{array}$ & $\begin{array}{l}\text { Preserved structure and function } \\
\text { after intravitreal rotenone } \\
\text { injection (rat) }\end{array}$ & $\begin{array}{l}\text { Leber's } \\
\text { hereditary optic } \\
\text { neuropathy }\end{array}$ & Rojas et $\mathrm{al}^{49}$ \\
\hline GaAIAs LED & $670 \mathrm{~nm}$ & $16 \mathrm{~J} / \mathrm{cm}^{2}$ in four fractions & $\begin{array}{l}\text { Preserved structure and function } \\
\text { after laser retinal photocoagulation } \\
\text { (monkey) }\end{array}$ & $\begin{array}{l}\text { Laser-induced } \\
\text { retinal injury }\end{array}$ & Eells et $\mathrm{al}{ }^{76}$ \\
\hline GaAIAs LED & $670 \mathrm{~nm}$ & $\begin{array}{l}50 \mathrm{~mW} / \mathrm{cm}^{2}, 20 \mathrm{~J} / \mathrm{cm}^{2} \\
\text { in five fractions }\end{array}$ & $\begin{array}{l}\text { Preserved structure in the } \\
\mathrm{P} 23 \mathrm{H}-3 \text { rat (rat) }\end{array}$ & $\begin{array}{l}\text { Retinitis } \\
\text { pigmentosa }\end{array}$ & Eells et $\mathrm{al}{ }^{76}$ \\
\hline GaAIAs LED & $670 \mathrm{~nm}$ & $\begin{array}{l}50 \mathrm{~mW} / \mathrm{cm}^{2}, 360 \mathrm{~J} / \mathrm{cm}^{2} \\
\text { in four fractions }\end{array}$ & $\begin{array}{l}\text { Preserved structure and function } \\
\text { after phototoxicity (rat) }\end{array}$ & $\begin{array}{l}\text { Light-induced } \\
\text { retinal damage }\end{array}$ & Qu et al, ${ }^{73}$ \\
\hline ILPD & $904 \mathrm{~nm}$ & $\begin{array}{l}4500 \mathrm{~mW} / \mathrm{cm}^{2}, 45,000 \\
\mathrm{~J} / \mathrm{m} 2, \text { pulsed at } 3 \mathrm{MHz}\end{array}$ & $\begin{array}{l}\text { Improved function in an 86-year- } \\
\text { old man with macular } \\
\text { degeneration (human) }\end{array}$ & $\begin{array}{l}\text { Age-related } \\
\text { macular } \\
\text { degeneration }\end{array}$ & $\begin{array}{l}\text { Rodriguez-Santana } \\
\text { et al, }{ }^{77}\end{array}$ \\
\hline
\end{tabular}

Abbreviations: GaAIAs LED, Gallium-Aluminum-Arsenide light-emitting diode; He-Ne, Helium-Neon; IPLD, intense pulsed light device.

spectrum. ${ }^{78}$ Photons at wavelengths between $630 \mathrm{~nm}$ and $800 \mathrm{~nm}$ have been shown to travel up to $28 \mathrm{~mm}$ even in layers of tissues with relatively low transparencies such as skin, connective tissue, muscle, bone, and spinal cord, with about $6 \%$ of the total energy density being detectable at the ventral surface of a living rat. ${ }^{42,80} \mathrm{In}$ gray matter, red and near-infrared light penetration is governed by Beer-Lambert law, with the optical power decaying up to $80 \%$ at $1 \mathrm{~mm}$ from the surface. ${ }^{81}$ However, at this depth in solid organs, the actual power density of near-infrared light has been estimated to be up to three times higher than the power at the incident surface due to backscattering and constructive interference. ${ }^{82}$ As the light travels into the tissue, its intensity decreases due to absorption and scattering. Penetration of light into tissues depends not only on the wavelength but also on the optical properties of the target tissue. The maximal penetration of light in the gray and white matter of the brain occurs at wavelengths in the near-infrared spectrum. ${ }^{81}$ It has been shown that within the visible and near-infrared spectral range, white matter in both the central and peripheral nervous systems reflects most of the incident power and shows a low level of absorption and a short penetration depth. ${ }^{83}$ In contrast, the transmittance of the gray matter is approximately twice as high as that of the white matter (Figure 2). Finally, the penetration of light is not only contingent on the wavelength or the specific tissue, but significant interspecies differences in penetration have also been detected. For example, at $850 \mathrm{~nm}$, the penetration of energy in humans is almost three times higher than that in the mouse cortex.$^{81}$ While the cause of this significant difference of light-tissue interaction can be explained by differences in water and protein content, this observation has obvious translational implications that should be considered when LLLT data generated in animal models is applied to humans. Finally, the delivery modality of LLLT is also relevant to transcranial in vivo applications (Table 1). For example, noncontact delivery modalities with LEDs allow exposure of extensive surfaces, including whole-body treatments. LEDs montages can potentially be built with ergonomic considerations for whole-head and whole-body LLLT in humans. In contrast, contact modalities combined with laser sources may be ideal when localized energy delivery is needed. This may be advantageous for boosting cell functions in specific nodes within dysfunctional neural networks in which connectivity could be otherwise impaired with broad irradiation. Similarly, localized transcranial LLLT may be of use for neuroprotection of healthy tissue adjacent to tumor sites after resection, without risk of inducing photobiomodulation of residual tumor. The eyes always need to be appropriately protected from laser light in transcranial applications.

Rojas et $\mathrm{al}^{49}$ were the first to demonstrate that upon transcranial delivery in vivo, LLLT induces whole-brain metabolic and antioxidant beneficial effects, as measured by increases in cytochrome oxidase and superoxide dismutase activities. Increases in cerebral blood flow induced by LLLT have also been observed in humans when applied transcranially. ${ }^{84}$ It is possible that these effects are related to a number of neuroprotective and function-enhancing effects that have been observed with the use of LLLT 
in vivo. The data presented below shows proof-of-principle that LLLT may be used in the treatment of neurovascular, neurodegenerative, and psychiatric disorders featuring impairments in energy metabolism. Of note, LLLT given transcranially at energy densities shown to exert beneficial effects have been also shown to induce no histological or behavioral adverse effects. Preclinical data on the use of LLLT to stimulate the brain in vivo support that this technology is safe. Adverse behavioral effects can be induced with massive energy densities 100 times higher than those observed to induce beneficial effects, but even the adverse effects of high doses can be attenuated if the total energy is delivered using intermittent energy pulses. ${ }^{85}$

\section{Stroke and neurotrauma}

Compelling evidence of the in vivo neuroprotective effects of LLLT against transient ischemia has been provided by recent studies conducted by Uozumi et al; ${ }^{86}$ LLLT delivered transcranially was able to increase cerebral blood flow. LLLT was given at $808 \mathrm{~nm}$ and $0.8 \mathrm{~W} / \mathrm{cm}^{2}, 1.6 \mathrm{~W} / \mathrm{cm}^{2}$, and $3.2 \mathrm{~W} /$ $\mathrm{cm}^{2}$ for 45 minutes over an exposure field of $3 \mathrm{~mm}$ in one hemisphere. Compared to sham subjects, the cerebral blood flow increased $30 \%$ with $1.6 \mathrm{~W} / \mathrm{cm}^{2}$, whereas the lowest and highest power densities were less efficient. The increases in cerebral blood flow were accompanied by a significant increase in nitric oxide production. Treating subjects with the nitric oxide synthase inhibitor NG-nitro-L-arginine methyl ester hydrochloride inhibited the increases in cerebral blood flow induced by LLLT. Similarly, the late increase in cerebral blood flow elicited by LLLT was attenuated by inhibition of glutamatergic transmission with the N-Methyl-D-aspartic acid competitive antagonist MK-801. These observations support that the increases in blood flow are secondary to an increased production of nitric oxide and related to neuronal activation. The neuroprotective effects of LLLT against decreased cerebrovascular perfusion in vivo were also demonstrated using a model of bilateral common carotid artery occlusion in mice. They showed that near-infrared treatment for 15-45 minutes increased local cerebral blood flow by $30 \%$ in this mouse model of stroke. Remarkably, the cerebral blood flow in conditions of normal and decreased perfusion was increased in both the treated and nontreated hemispheres. Also, subjects pretreated with LLLT showed improved residual cerebral blood flow during the period of occlusion, with stable body temperature, heart rate, and respiratory rates. Finally, the transient cerebral ischemia induced by carotid occlusion produced cell death in $84 \%$ of cells in the CA1 field of the hippocampus and in $27 \%$ of cortical neurons 96 hours after the insult. In contrast, LLLT had a significant neuroprotective effect by decreasing the number of apoptotic cells to only $44 \%$ and $8 \%$ in the hippocampus and cortex, respectively, after transient ischemia. Of note, these experiments showed that LLLT and neuroprotection against transient ischemia were not associated with increased expression of nitric oxide synthase. This suggests that the observed increases in nitric oxide concentrations were the product of nitric oxide synthase-independent mechanisms. Mechanisms of nitric oxide synthase-independent nitric oxide formation include reduction of nitrite by xanthine oxidase or cytochromes. As mentioned before, LLLT increases brain cytochrome oxidase expression in vivo ${ }^{49}$ and the activation of intracellular signals induced by LLLT seem to be contingent upon concentrations of nitric oxide. ${ }^{87}$

DeTaboada et $\mathrm{al}^{88}$ tested the neuroprotective effects of LLLT given 24 hours after stroke in a rat model of unilateral carotid artery occlusion that induces measurable motor neurological deficits. LLLT was given at $808 \mathrm{~nm}$ with an energy density of $0.9 \mathrm{~J} / \mathrm{cm}^{2}$ and power density of $7.5 \mathrm{~mW} /$ $\mathrm{cm}^{2}$ to the hemisphere ipsilateral to the lesion, contralateral to the lesion, or both. At 28 days, LLLT enhanced the neurological recovery by an average of $38 \%,{ }^{88}$ and these effects were associated with increased neuronal proliferation and migration in the subventricular zone. ${ }^{89}$

LLLT has also been effective at improving the behavioral deficits in a rabbit model of embolic strokes induced by microclot injection directly into the common carotid artery. ${ }^{90}$ LLLT was initiated 1 hour, 3 hours, 6 hours, or 24 hours after embolization. Treatment was given at $808 \mathrm{~nm}$ with a $2-\mathrm{cm}$ probe and a power density of $7.5 \mathrm{~mW} / \mathrm{cm}^{2}$ for 10 minutes. LLLT significantly improved the deficits compared with controls when measured 24 hours after treatment. This effect was durable and observed 3 weeks after embolization. No beneficial effect was observed when LLLT was started 24 hours after embolization. Beneficial effects were observed after a window of 6 hours when LLLT was given not as a continuous wave, but as a pulse with frequencies of 300 microseconds at $1 \mathrm{kHz}$ or 2 microseconds at $100 \mathrm{~Hz}{ }^{91}$ Furthermore, whereas thrombolytic therapy increased the incidence of hemorrhage compared to controls, LLLT decreased the incidence of hemorrhage induced by thrombolytic therapy by $30 \%$ and had no effect on hemorrhage rate when given alone. ${ }^{92}$ In this model of embolic stroke, there was $45 \%$ decreased ATP content in the ischemic cortex compared to naive rabbits 3 hours after embolization. LLLT given as a continuous pulse resulted in a $41 \%$ increase in cortical ATP content compared to the 
sham embolized group and in an absolute increase in ATP content of $22.5 \%$ compared to naive rabbits. This effect was maximized when a higher LLLT energy was delivered as a pulse. When five and 35 times more energy was delivered, the amount of cortical ATP increased $157 \%$ and $221 \%$ compared to the sham embolized group, respectively. ${ }^{93}$

Encouraging results from a recent clinical study suggest that LLLT is safe and might be effective in the treatment of ischemic stroke in humans. ${ }^{94}$ The NeuroThera Effectiveness and Safety Trial-1 tested the safety and effectiveness of LLLT at $808 \mathrm{~nm}$ to improve the 90 -day outcomes in ischemic stroke given within 24 hours from stroke onset. This study included 120 patients with National Institutes of Health Stroke Scale (NIHSS) scores of 7-22. Patients receiving thrombolytic therapy were excluded. The primary outcome measure was a complete recovery at day 90 (NIHSS 0-1) or a decrease in NIHSS score of at least nine points. Median time to treatment was 18 hours. Among the 79 treated patients, 38\% achieved both a final NIHSS score of $0-1$ and improved by more than nine points, $20 \%$ had only a more than nine-point improvement, $11 \%$ obtained a final score of $0-1$ without improving by more than nine, and $30 \%$ achieved neither end point. Among the 41 control patients the corresponding proportions were $29 \%, 7 \%, 15 \%$, and $49 \%$. The mortality rates and adverse effects were similar in the treated and control groups. ${ }^{94}$ However, a larger follow-up multicenter double-blind study that randomized 660 patients to LLLT or sham showed no significant difference in favorable outcomes, although favorable trends were observed in the LLLT group for less disability at 90 days. In this second study, LLLT seemed to benefit patients with lower NIHSS scores at baseline. ${ }^{95}$

Together, these experiments provide in vivo evidence that the effects of LLLT on cytochrome oxidase and nitric oxide play a major role in the neuroprotective action of LLLT against ischemia and possibly other metabolic insults, such as the LLLT benefits found after traumatic brain injury in animals ${ }^{96}$ and humans. ${ }^{97}$ Current clinical trials are investigating the beneficial effects of transcranial LLLT in the rehabilitation of hemiplegic patients after ischemic stroke. ${ }^{98}$ Further clinical research is expected to improve the understanding of LLLT effects in stroke and neurotrauma patients.

\section{Parkinson's disease}

The protective effects of LLLT have also been demonstrated in paradigms of neurodegeneration. Shaw et $\mathrm{al}^{99}$ showed that LLLT has a potential application in the treatment of Parkinson's disease. This group tested the neuroprotective effects if LLLT in a mouse in vivo model of dopaminergic degeneration induced by the neurotoxin 1-methyl-4phenyl-1,2,3,6-tetrahydropyridine (MPTP). LLLT was given 15 minutes after MPTP intraperitoneal injection at $670 \mathrm{~nm}$ and $40 \mathrm{~mW} / \mathrm{cm}^{2}$. A total dose of $2 \mathrm{~J} / \mathrm{cm}^{2}$ was delivered in four fractions of 9 seconds evenly spaced over 30 hours. Approximately $10 \%$ of the power density reaching the skull reached the brain. Two days after MPTP injection, there was a $45 \%$ reduction in the number of dopaminergic cells in the substantia nigra compared to saline-treated controls. LLLT completely prevented the loss of dopaminergic cells in the substantia nigra. When the dose of MPTP was doubled, there was an even more profound decrease in dopaminergic cells of $60 \%$. However, LLLT limited the neurodegeneration induced by this higher MPTP dose to only 30\% compared to control. This study not only offered evidence that LLLT induces neuroprotective effects against dopaminergic toxins in vivo, but that its effects reach structures deep in the brain.

\section{Cognition and emotional states}

Among the most fascinating experimental applications of LLLT are those related to enhancement of normal brain function and the treatment of memory loss and mood disorders. Cognitive impairment and neurodegeneration associated with dementia have been shown to have regional brain metabolic deficits in early stages of the neurodegenerative process. For example, early decreases in brain metabolic activity can be detected in patients at risk of developing Alzheimer's disease, especially reductions in cytochrome oxidase activity. ${ }^{100,101}$ Similarly, the phenotypic expression of mood disorders such as major depression and post-traumatic stress disorder have been shown to be associated with decreased metabolic capacity in prefrontal brain regions, ${ }^{102}$ and electrical stimulation of prefrontal cortex has antidepressant effects. ${ }^{103}$ LLLT is expected to enhance the metabolic capacity in those regions showing functional deficits, thus increasing the functional connectivity of the networks involved in the expression of a particular phenotype. This is in addition to the potential neuroprotective effects that LLLT may have in tissue susceptible to neurodegeneration. Michalikova et al ${ }^{104}$ demonstrated improvement in working memory using LLLT in middle-aged mice tested in an appetitive spatial navigation task. Such memory improvements occurred in the absence of nonspecific effects on exploratory activity or anxiety responses. This study used LLLT at $1072 \mathrm{~nm}$ that was given in 10 fractions, one fraction per day. However, no other LLLT dose parameters such as power density or energy density were reported. Mice treated with LLLT showed higher latencies to make a choice, but with improved correct choice rates. Indeed 
Table 3 Beneficial in vivo transcranial effects of low-level light therapy on the brain

\begin{tabular}{|c|c|c|c|c|c|}
\hline Source & Wavelength & Dose & Effect & Relevance & Reference \\
\hline Laser & $808 \mathrm{~nm}$ & $1.6 \mathrm{~W} / \mathrm{cm}^{2}, 4320 \mathrm{~J} / \mathrm{cm}^{2}$ & $\begin{array}{l}\text { Increased cerebral blood flow and decreased } \\
\text { hippocampal and cortical neuronal death } \\
\text { after unilateral BCCAO (mouse) }\end{array}$ & Anoxic brain injury & Uozumi et al, ${ }^{86}$ \\
\hline Laser & $808 \mathrm{~nm}$ & $\begin{array}{l}7.5 \mathrm{~mW} / \mathrm{cm}^{2}, 0.9 \mathrm{~J} / \mathrm{cm}^{2} \text {, } \\
2 \text { minutes per point }\end{array}$ & $\begin{array}{l}\text { Improved neurological recovery, increased } \\
\text { subventricular neural proliferation after } \\
\text { MCAO (rat) }\end{array}$ & $\begin{array}{l}\text { Atherothrombotic } \\
\text { stroke }\end{array}$ & $\begin{array}{l}\text { DeTaboada et al, }{ }^{88} \\
\text { Oron et al, }{ }^{89}\end{array}$ \\
\hline Laser & $808 \mathrm{~nm}$ & $\begin{array}{l}25 \mathrm{~mW} / \mathrm{cm}^{2}, 15,000 \mathrm{~J} / \mathrm{cm}^{2} \text {, } \\
\text { continuous }\end{array}$ & $\begin{array}{l}\text { Improved motor function and reduction } \\
\text { in effective clot dose for stroke } 3 \text { hours } \\
\text { after clot injection (rabbit) }\end{array}$ & Embolic stroke & Lapchak et al, ${ }^{90}$ \\
\hline Laser & $808 \mathrm{~nm}$ & $\begin{array}{l}25 \mathrm{~mW} / \mathrm{cm}^{2}, 15,000 \mathrm{~J} / \mathrm{cm}^{2}, \\
\text { pulsed at I } \mathrm{KHz}\end{array}$ & $\begin{array}{l}\text { Increased cortical ATP, decreased effective } \\
\text { clot dose for stroke } 6 \text { hours after } \\
\text { clot injection (rabbit) }\end{array}$ & Embolic stroke & Lapchak et al ${ }^{91-93}$ \\
\hline Laser & $808 \mathrm{~nm}$ & I J/ $\mathrm{cm}^{2}$ per point & $\begin{array}{l}\text { Improved clinical outcome at } 90 \text { days } \\
\text { after ischemic stroke (human) }\end{array}$ & Ischemic stroke & Lampl et al, ${ }^{94}$ \\
\hline Laser & $808 \mathrm{~nm}$ & $\begin{array}{l}10 \text { or } 20 \mathrm{~mW} / \mathrm{cm}^{2} \text {, } \\
\text { I. } 2-2.4 \mathrm{~J} / \mathrm{cm}^{2} \text {, single point } \\
\text { for } 2 \text { minutes }\end{array}$ & $\begin{array}{l}\text { Improved motor behavior } 5 \text { days after } \\
\text { closed-head injury, and decreased brain } \\
\text { lesion size from } 12.1 \% \text { to } 1.4 \% \text { at } \\
28 \text { days after injury (mouse) }\end{array}$ & $\begin{array}{l}\text { Traumatic brain } \\
\text { injury (acute) }\end{array}$ & Oron et al, ${ }^{96}$ \\
\hline LED & $\begin{array}{l}633 \text { and } 870 \mathrm{~nm} \\
\text { LED cluster }\end{array}$ & $\begin{array}{l}22.2 \mathrm{~mW} / \mathrm{cm}^{2}, 13.3 \mathrm{~J} / \mathrm{cm}^{2} \text {, } \\
10 \text { minutes per placement }\end{array}$ & $\begin{array}{l}\text { Improved cognition of two patients } \\
\text { with chronic mild traumatic brain injury } \\
\text { after } 2-4 \text { months of treatment (human) }\end{array}$ & $\begin{array}{l}\text { Traumatic brain } \\
\text { injury (chronic) }\end{array}$ & Naeser et al, ${ }^{97}$ \\
\hline Laser & $670 \mathrm{~nm}$ & $\begin{array}{l}40 \mathrm{~mW} / \mathrm{cm}^{2}, 2 \mathrm{~J} / \mathrm{cm}^{2} \text { in } \\
\text { four fractions }\end{array}$ & $\begin{array}{l}\text { Reduction in substantia nigra dopaminergic } \\
\text { cell loss after MPTP toxicity (mouse) }\end{array}$ & Parkinson's disease & Shaw et al, ${ }^{99}$ \\
\hline Laser & $1072 \mathrm{~nm}$ & 6 minutes $\times 10$ days & $\begin{array}{l}\text { Improved acquisition of working memory } \\
\text { for spatial navigation in middle-aged } \\
\text { mice (mouse) }\end{array}$ & $\begin{array}{l}\text { Mild cognitive } \\
\text { impairment, } \\
\text { Alzheimer's disease }\end{array}$ & $\begin{array}{l}\text { Michalikova } \\
\text { et al, }{ }^{104}\end{array}$ \\
\hline LED & $810 \mathrm{~nm}$ & $250 \mathrm{~mW} / \mathrm{cm}^{2}, 60 \mathrm{~J} / \mathrm{cm}^{2}$ & $\begin{array}{l}\text { Decreased depression scores, increased } \\
\text { prefrontal blood flow (human) }\end{array}$ & $\begin{array}{l}\text { Depression, } \\
\text { prefrontal functions }\end{array}$ & Schiffer et al, ${ }^{84}$ \\
\hline
\end{tabular}

Abbreviations: ATP, adenosine triphosphate; BCCAO, bilateral common carotid artery occlusion; LED, light-emitting device; MCAO, middle cerebral artery occlusion; MPTP, I-methyl-4-phenyl-1,2,3,6-tetrahydropyridine.

the memory performance of LLLT-treated middle-aged mice became comparable to that of young mice. These data support that LLLT could be particularly useful at facilitating acquisition of a working memory task in conditions in which information processing speed is impaired, such as aging.

Experimental data in humans also support that LLLT may be effective in the treatment of cognitive and emotional disorders in humans. A pilot study showed that LLLT applied transcranially to the forehead was able to increase frontal cortex blood flow and induce a $63 \%$ reduction in depression scores in a group of patients with major depression. Beneficial antidepressant effects were seen 2 weeks and 4 weeks after a single treatment. LLLT was given in one fraction at $810 \mathrm{~nm}$, power density of $250 \mathrm{~mW} / \mathrm{cm}^{2}{ }^{84}$ Additionally, LLLT to the forehead and scalp with an LED cluster at $633 \mathrm{~nm}$ and $870 \mathrm{~nm}$ has been reported to improve and maintain attention, executive function, and memory in two patients with chronic traumatic brain injury. ${ }^{97}$ LLLT with a power density of $22.2 \mathrm{~mW} / \mathrm{cm}^{2}$ was applied for 10 minutes per placement weekly for 2 months or daily for 4 months prior to testing of the cognitive effects, and these patients have continued daily treatment at home for up to 5.5 years. Remarkably, no side effects have been reported so far with the use of LLLT in humans (Table 3).

Research on the application of LLLT for the improvement of brain function is expected to increase in the following years. The use of this technology will benefit from further studies looking for optimal LLLT parameters and treatment protocols. Current preclinical data support the feasibility of formal randomized placebo-controlled trials, and these will likely be developed in the near future.

\section{Conclusion}

LLLT or photobiomodulation refers to the use of low-power and high-fluence light from lasers or LEDs in the red to near-infrared wavelengths to modulate a biological function. Cytochrome oxidase is the primary photoacceptor of LLLT with beneficial eye and brain effects since this mitochondrial enzyme is crucial for oxidative energy metabolism, and neurons depend on cytochrome oxidase to produce their metabolic energy. Photon-induced redox mechanisms in cytochrome oxidase cause other primary and secondary 
hormetic responses in neurons that may be beneficial for neurotherapeutic purposes. Beneficial in vivo effects of LLLT on the eye have been found in optic nerve trauma, methanol intoxication, optic neuropathy, retinal injury, retinitis pigmentosa, phototoxicity, and age-related macular degeneration. Beneficial in vivo transcranial effects of LLLT on the brain have been observed in anoxic brain injury, atherothrombotic stroke, embolic stroke, ischemic stroke, acute traumatic brain injury, chronic traumatic brain injury, neurodegeneration, age-related memory loss, and cognitive and mood disorders. No adverse side effects have been reported in these beneficial applications of LLLT in animals and humans. The authors conclude that LLLT is a safe and beneficial approach, based on scientifically sound mechanisms of action of red to nearinfrared light on cytochrome oxidase, with neurotherapeutic promise for a wide range of ophthalmological, neurological, and psychological conditions.

\section{Disclosure}

The authors report no conflicts of interest in this work.

\section{References}

1. Hashmi JT, Huang YY, Osmani BZ, Sharma SK, Naeser MA, Hamblin MR. Role of low-level laser therapy in neurorehabilitation. Pm R. 2010;2(12 Suppl 2):S292-S305.

2. Sommer AP, Pinheiro AL, Mester AR, Franke RP, Whelan HT. Biostimulatory windows in low-intensity laser activation: lasers, scanners, and NASA's light-emitting diode array system. J Clin Laser Med Surg. 2001;19(1):29-33.

3. Karu TI. Mitochondrial signaling in mammalian cells activated by red and near-IR radiation. Photochem Photobiol. 2008;84(5):1091-1099.

4. Morton CA, McKenna KE, Rhodes LE. Guidelines for topical photodynamic therapy: update. Br J Dermatol. 2008;159(6):1245-1266.

5. Fork RL. Laser stimulation of nerve cells in Aplysia. Science. 1971;171(974):907-908.

6. Hamblin MR, Demidova TN. Mechanisms of low level light therapy. Proc SPIE. 2006;6140:1-12.

7. Sutherland JC. Biological effects of polychromatic light. Photochem Photobiol. 2002;76(2):164-170.

8. Eells JT, Wong-Riley MT, VerHoeve J, et al. Mitochondrial signal transduction in accelerated wound and retinal healing by near-infrared light therapy. Mitochondrion. 2004;4(5-6):559-567.

9. Wong-Riley MT, Liang HL, Eells JT, et al. Photobiomodulation directly benefits primary neurons functionally inactivated by toxins: role of cytochrome c oxidase. J Biol Chem. 2005;280(6):4761-4771.

10. Calabrese EJ, Stanek EJ 3rd, Nascarella MA, Hoffmann GR. Hormesis predicts low-dose responses better than threshold models. Int J Toxicol. 2008;27(5):369-378.

11. Yu W, Naim JO, Lanzafame RJ. The effect of laser irradiation on the release of bFGF from 3 T3 fibroblasts. Photochem Photobiol. 1994;59(2):167-170.

12. Eells JT, Henry MM, Summerfelt P, et al. Therapeutic photobiomodulation for methanol-induced retinal toxicity. Proc Natl Acad Sci U S A. 2003;100(6):3439-3444

13. Lectures N. Physiology or Medicine 1903 - Presentation Speech. In: Nobelprize.org, editor; 2011. Available from: http://www.nobelprize. org/nobel_prizes/medicine/laureates/1903/press.html. Accessed October 6, 2011.
14. Santana-Blank L, Rodriguez-Santana E, Santana-Rodriguez K. Theoretic, experimental, clinical bases of the water oscillator hypothesis in near-infrared photobiomodulation. Photomed Laser Surg. 2010;28 Suppl 1:S41-S52.

15. Chiaravalloti F, Gross L, Rieder KH, et al. A rack-and-pinion device at the molecular scale. Nat Mater. 2007;6(1):30-33.

16. Karu TI, Pyatibrat LV, Moskvin SV, Andreev S, Letokhov VS. Elementary processes in cells after light absorption do not depend on the degree of polarization: implications for the mechanisms of laser phototherapy. Photomed Laser Surg. 2008;26(2):77-82.

17. Benarroch EE. The melanopsin system: Phototransduction, projections, functions, and clinical implications. Neurology. 2011;76(16):1422-1427.

18. Bellingham J, Chaurasia SS, Melyan Z, et al. Evolution of melanopsin photoreceptors: discovery and characterization of a new melanopsin in nonmammalian vertebrates. PLoS Biol. 2006;4(8):e254.

19. Okano T, Yoshizawa T, Fukada Y. Pinopsin is a chicken pineal photoreceptive molecule. Nature. 1994;372(6501):94-97.

20. Karu TI. Molecular mechanism of the therapeutic effect of low intensity laser radiation. Lasers Life Sci. 1988;2:53-74.

21. Karu T. Primary and secondary mechanisms of action of visible to near-IR radiation on cells. J Photochem Photobiol B. 1999;49(1):1-17.

22. Kubota S, Yang JT. Bis[cyclo(histidylhistidine)]copper(II) complex that mimicks the active center of superoxide dismutase has its catalytic activity. Proc Natl Acad Sci USA. 1984;81(11):3283-3286.

23. Kato M, Shinizawa K, Yoshikawa S. Cythochrome oxidase is a possible photoreceptor in mitochondria. Photobiochem Photobiophys. 1981;2:263-269.

24. Karu T. Mechanisms of low-power laser light action on cellular level. Proc SPIE. 2000;4159:1-17.

25. Karu T. Laser biostimulation: a photobiological phenomenon. J Photochem Photobiol B. 1989;3(4):638-640.

26. Pastore D, Greco M, Passarella S. Specific helium-neon laser sensitivity of the purified cytochrome c oxidase. Int J Radiat Biol. 2000;76(6):863-870.

27. Yamanaka T, Fukumori Y, Numata M, Yamazaki T. The variety of molecular properties of bacterial cytochromes containing heme a. Ann NY Acad Sci. 1988;550:39-46.

28. Wong-Riley M. Energy metabolism of the visual system. Eye and Brain. 2010;2:99-116.

29. Hatefi Y. The mitochondrial electron transport and oxidative phosphorylation system. Annu Rev Biochem. 1985;54:1015-1069.

30. Sakata JT, Crews D, Gonzalez-Lima F. Behavioral correlates of differences in neural metabolic capacity. Brain Res Brain Res Rev. 2005;48(1):1-15.

31. Wong-Riley MT. Cytochrome oxidase: an endogenous metabolic marker for neuronal activity. Trends Neurosci. 1989;12(3):94-101.

32. Karu TI, Pyatibrat LV, Kalendo GS, Esenaliev RO. Effects of monochromatic low-intensity light and laser irradiation on adhesion of HeLa cells in vitro. Lasers Surg Med. 1996;18(2):171-177.

33. Karu TI, Pyatibrat LV, Kolyakov SF, Afanasyeva NI. Absorption measurements of cell monolayers relevant to mechanisms of laser phototherapy: reduction or oxidation of cytochrome c oxidase under laser radiation at $632.8 \mathrm{~nm}$. Photomed Laser Surg. 2008;26(6):593-599.

34. Huang YY, Chen AC, Carroll JD, Hamblin MR. Biphasic dose response in low level light therapy. Dose Response. 2009;7(4):358-383.

35. Karu T. Mitochondrial mechanisms of photobiomodulation in context of new data about multiple roles of ATP. Photomed Laser Surg. 2010;28(2):159-160.

36. Wong-Riley MT, Bai X, Buchmann E, Whelan HT. Light-emitting diode treatment reverses the effect of TTX on cytochrome oxidase in neurons. Neuroreport. 2001;12(14):3033-3037.

37. Hayworth CR, Rojas JC, Padilla E, Holmes GM, Sheridan EC, Gonzalez-Lima F. In vivo low-level light therapy increases cytochrome oxidase in skeletal muscle. Photochem Photobiol. 2010;86(3):673-680.

38. Liang HL, Whelan HT, Eells JT, et al. Photobiomodulation partially rescues visual cortical neurons from cyanide-induced apoptosis. Neuroscience. 2006;139(2):639-649. 
39. Byrnes KR, Wu X, Waynant RW, Ilev IK, Anders JJ. Low power laser irradiation alters gene expression of olfactory ensheathing cells in vitro. Lasers Surg Med. 2005;37(2):161-171.

40. Liang HL, Whelan HT, Eells JT, Wong-Riley MT. Near-infrared light via light-emitting diode treatment is therapeutic against rotenone- and 1-methyl-4-phenylpyridinium ion-induced neurotoxicity. Neuroscience. 2008;153(4):963-974.

41. Ying R, Liang HL, Whelan HT, Eells JT, Wong-Riley MT. Pretreatment with near-infrared light via light-emitting diode provides added benefit against rotenone- and $\mathrm{MPP}^{+}$-induced neurotoxicity. Brain Res. 2008;1243:167-173.

42. Byrnes KR, Waynant RW, Ilev IK, et al. Light promotes regeneration and functional recovery and alters the immune response after spinal cord injury. Lasers Surg Med. 2005;36(3):171-185.

43. Chow RT, Johnson MI, Lopes-Martins RA, Bjordal JM. Efficacy of low-level laser therapy in the management of neck pain: a systematic review and meta-analysis of randomised placebo or active-treatment controlled trials. Lancet. 2009;374(9705):1897-1908.

44. Duan R, Zhu L, Liu TC, et al. Light emitting diode irradiation protect against the amyloid beta 25-35 induced apoptosis of PC12 cell in vitro. Lasers Surg Med. 2003;33(3):199-203.

45. Wollman Y, Rochkind S. In vitro cellular processes sprouting in cortex microexplants of adult rat brains induced by low power laser irradiation. Neurol Res. 1998;20(5):470-472.

46. Van Breugel HH, Bar PR. He-Ne laser irradiation affects proliferation of cultured rat Schwann cells in a dose-dependent manner. J Neurocytol. 1993;22(3):185-190.

47. Rochkind S, Vogler I, Barr-Nea L. Spinal cord response to laser treatment of injured peripheral nerve. Spine (Phila Pa 1976). 1990;15(1):6-10.

48. Anders JJ, Borke RC, Woolery SK, Van de Merwe WP. Low power laser irradiation alters the rate of regeneration of the rat facial nerve. Lasers Surg Med. 1993;13(1):72-82.

49. Rojas JC, Lee J, John JM, Gonzalez-Lima F. Neuroprotective effects of near-infrared light in an in vivo model of mitochondrial optic neuropathy. J Neurosci. 2008;28(50):13511-13521.

50. Jester JV, Moller-Pedersen T, Huang J, et al. The cellular basis of corneal transparency: evidence for 'corneal crystallins'. J Cell Sci. 1999;112 (Pt 5):613-622.

51. Ames A 3rd, Li YY, Heher EC, Kimble CR. Energy metabolism of rabbit retina as related to function: high cost of $\mathrm{Na}^{+}$transport. $J$ Neurosci. 1992;12(3):840-853.

52. Astrup J, Sorensen PM, Sorensen HR. Oxygen and glucose consumption related to $\mathrm{Na}^{+}-\mathrm{K}^{+}$transport in canine brain. Stroke. 1981;12(6):726-730.

53. Kann O, Kovacs R. Mitochondria and neuronal activity. Am J Physiol Cell Physiol. 2007;292(2):C641-657.

54. Beretta S, Wood JP, Derham B, et al. Partial mitochondrial complex I inhibition induces oxidative damage and perturbs glutamate transport in primary retinal cultures. Relevance to Leber Hereditary Optic Neuropathy (LHON). Neurobiol Dis. 2006;24(2):308-317.

55. Levin LA, Peeples P. History of neuroprotection and rationale as a therapy for glaucoma. Am J Manag Care. 2008;14(1 Supp1):S11-S14.

56. Rojas JC, Gonzalez-Lima F. Mitochondrial optic neuropathy: in vivo model of neurodegeneration and neuroprotective strategies. Eye and Brain. 2010;2:21-37.

57. Abu-Amero KK, Bosley TM. Mitochondrial abnormalities in patients with LHON-like optic neuropathies. Invest Ophthalmol Vis Sci. 2006;47(10):4211-4220.

58. Carelli V, La Morgia C, Valentino ML, Barboni P, Ross-Cisneros FN, Sadun AA. Retinal ganglion cell neurodegeneration in mitochondrial inherited disorders. Biochim Biophys Acta. 2009;1787(5):518-528.

59. Fortuna F, Barboni P, Liguori R, et al. Visual system involvement in patients with Friedreich's ataxia. Brain. 2009;132(Pt 1):116-123.

60. Iseri PK, Altinas O, Tokay T, Yuksel N. Relationship between cognitive impairment and retinal morphological and visual functional abnormalities in Alzheimer disease. J Neuroophthalmol. 2006;26(1):18-24.
61. Danesh-Meyer HV, Birch H, Ku JY, Carroll S, Gamble G. Reduction of optic nerve fibers in patients with Alzheimer disease identified by laser imaging. Neurology. 2006;67(10):1852-1854.

62. Hinton DR, Sadun AA, Blanks JC, Miller CA. Optic-nerve degeneration in Alzheimer's disease. N Engl J Med. 1986;315(8):485-487.

63. Chalmers RM, Schapira AH. Clinical, biochemical and molecular genetic features of Leber's hereditary optic neuropathy. Biochim Biophys Acta. 1999;1410(2):147-158.

64. BoritA. Leigh's necrotizing encephalomyelopathy. Neuro-ophthalmological abnormalities. Arch Ophthalmol. 1971;85(4):438-442.

65. DiMauro S. Mitochondrial encephalomyopathies: back to Mendelian genetics. Ann Neurol. 1999;45(6):693-694.

66. Carelli V, Ross-Cisneros FN, Sadun AA. Optic nerve degeneration and mitochondrial dysfunction: genetic and acquired optic neuropathies. Neurochem Int. 2002;40(6):573-584.

67. Chinnery PF, Howell N, Lightowlers RN, Turnbull DM. Molecular pathology of MELAS and MERRF. The relationship between mutation load and clinical phenotypes. Brain. 1997;120(Pt 10):1713-1721.

68. Hwang JM, Park HW, Kim SJ. Optic neuropathy associated with mitochondrial tRNA[Leu(UUR)] A3243G mutation. Ophthalmic Genet. 1997;18(2):101-105.

69. Casari G, De Fusco M, Ciarmatori S, et al. Spastic paraplegia and OXPHOS impairment caused by mutations in paraplegin, a nuclear-encoded mitochondrial metalloprotease. Cell. 1998;93(6): 973-983.

70. Tranebjaerg L, Hamel BC, Gabreels FJ, Renier WO, Van Ghelue M. A de novo missense mutation in a critical domain of the X-linked DDP gene causes the typical deafness-dystonia-optic atrophy syndrome. Eur J Hum Genet. 2000;8(6):464-467.

71. Schwartz M, Doron A, Erlich M, et al. Effects of low-energy He-Ne laser irradiation on posttraumatic degeneration of adult rabbit optic nerve. Lasers Surg Med. 1987;7(1):51-55.

72. Assia E, Rosner M, Belkin M, Solomon A, Schwartz M. Temporal parameters of low energy laser irradiation for optimal delay of post-traumatic degeneration of rat optic nerve. Brain Res. 1989;476(2):205-212.

73. Qu C, Cao W, Fan Y, Lin L. Near-infrared light protect the photoreceptor from light-induced damage in rats. In: Anderson RE, editor. Retinal Degenerative Diseases. New York, NY: Springer Science; 2010:365-374.

74. Albarracin R, Eells J, Valter K. Photobiomodulation protects the retina from light-induced photoreceptor degeneration. Invest Ophthalmol Vis Sci. 2011;52(6):3582-3592.

75. Natoli R, Zhu Y, Valter K, Bisti S, Eells J, Stone J. Gene and noncoding RNA regulation underlying photoreceptor protection: microarray study of dietary antioxidant saffron and photobiomodulation in rat retina. $\mathrm{Mol}$ Vis. 2010;16:1801-1822.

76. Eells J, DeSmet KD, Kirk DK, et al. Photobiomodulation for the treatment of retinal injury and retinal degenerative diseases. In: Waynant RW, Tata DB, editors. Proceedings of Light-Activated Tissue Regeneration and Therapy Conference. New York, NY: Springer Science; 2008:39-51

77. Rodriguez-Santana E, Reyes H, Santana-Rodriguez KE, Santana-Blank L. Photo-infrared pulsed biomodulation in age-related macular degeneration associated to neurological disease: one interventional case report and mini-review. J Chinese Clin Med. 2008;3:470-477.

78. Helm PJ, Ottersen OP, Nase G. Analysis of optical properties of the mouse cranium - implications for in vivo multi photon laser scanning microscopy. J Neurosci Methods. 2009;178(2):316-322.

79. Orihuela-Espina F, Leff DR, James DR, Darzi AW, Yang GZ. Quality control and assurance in functional near infrared spectroscopy (fNIRS) experimentation. Phys Med Biol. 2010;55(13):3701-3724.

80. Chance B, Nioka S, Kent J, et al. Time-resolved spectroscopy of hemoglobin and myoglobin in resting and ischemic muscle. Anal Biochem. 1988;174(2):698-707.

81. Abdo A, Sahin M. NIR light penetration depth in the rat peripheral nerve and brain cortex. Conf Proc IEEE Eng Med Biol Soc. 2007; 2007:1723-1725. 
82. Grimvlatov V, Rubinshtein A, Rubinshtein M. Spectral dosimetry in low light therapy. Proc SPIE. 2006;6140:1-11.

83. Eggert HR, Blazek V. Optical properties of human brain tissue, meninges, and brain tumors in the spectral range of 200 to $900 \mathrm{~nm}$. Neurosurgery. 1987;21(4):459-464.

84. Schiffer F, Johnston AL, Ravichandran C, et al. Psychological benefits 2 and 4 weeks after a single treatment with near infrared light to the forehead: a pilot study of 10 patients with major depression and anxiety. Behav Brain Funct. 2009;5:46.

85. Ilic S, Leichliter S, Streeter J, Oron A, DeTaboada L, Oron U. Effects of power densities, continuous and pulse frequencies, and number of sessions of low-level laser therapy on intact rat brain. Photomed Laser Surg. 2006;24(4):458-466.

86. Uozumi Y, Nawashiro H, Sato S, Kawauchi S, Shima K, Kikuchi M. Targeted increase in cerebral blood flow by transcranial near-infrared laser irradiation. Lasers Surg Med. 2010;42(6):566-576.

87. Karu T, Pyatibrat LV, Afanasyeva NI. Cellular effects of low power laser therapy can be mediated by nitric oxide. Laser Surg Med. 2005;36:307-314.

88. Detaboada L, Ilic S, Leichliter-Martha S, Oron U, Oron A, Streeter J. Transcranial application of low-energy laser irradiation improves neurological deficits in rats following acute stroke. Lasers Surg Med. 2006;38(1):70-73

89. Oron A, Oron U, Chen J, et al. Low-level laser therapy applied transcranially to rats after induction of stroke significantly reduces long-term neurological deficits. Stroke. 2006;37(10):2620-2624.

90. Lapchak PA, Wei J, Zivin JA. Transcranial infrared laser therapy improves clinical rating scores after embolic strokes in rabbits. Stroke. 2004;35(8):1985-1988.

91. Lapchak PA, Salgado KF, Chao CH, Zivin JA. Transcranial near-infrared light therapy improves motor function following embolic strokes in rabbits: an extended therapeutic window study using continuous and pulse frequency delivery modes. Neuroscience. 2007;148(4):907-914.

92. Lapchak PA, Han MK, Salgado KF, Streeter J, Zivin JA. Safety profile of transcranial near-infrared laser therapy administered in combination with thrombolytic therapy to embolized rabbits. Stroke. 2008;39(11):3073-3078.

93. Lapchak PA, De Taboada L. Transcranial near infrared laser treatment (NILT) increases cortical adenosine-5' ${ }^{\prime}$-triphosphate (ATP) content following embolic strokes in rabbits. Brain Res. 2010;1306:100-105.
94. LamplY, Zivin JA, Fisher M, et al. Infrared laser therapy for ischemic stroke: a new treatment strategy: results of the NeuroThera Effectiveness and Safety Trial-1 (NEST-1). Stroke. 2007;38(6):1843-1849.

95. Zivin JA, Albers GW, Bornstein N, et al. Effectiveness and safety of transcranial laser therapy for acute ischemic stroke. Stroke. 2009;40(4):1359-1364.

96. Oron A, Oron U, Streeter J, et al. Low-level laser therapy applied transcranially to mice following traumatic brain injury significantly reduces long-term neurological deficits. $J$ Neurotrauma. 2007;24(4):651-656.

97. Naeser MA, Saltmarche A, Krengel MH, Hamblin MR, Knight JA. Improved cognitive function after transcranial, light-emitting diode treatments in chronic, traumatic brain injury: two case reports. Photomed Laser Surg. 2010;29(5):351-358.

98. ClinicalTrials.gov. Transcranial laser therapy in the rehabilitation of hemiplegic patients from ischemic stroke (REHELA). March 3, 2011. Available from: http://clinicaltrials.gov/ct2/show/NCT01308216?ter $\mathrm{m}=\mathrm{NCT} 01308216 \&$ rank=1. Accessed September 23, 2011.

99. Shaw VE, Spana S, Ashkan K, et al. Neuroprotection of midbrain dopaminergic cells in MPTP-treated mice after near-infrared light treatment. J Comp Neurol. 2010;518(1):25-40.

100. Valla J, Berndt JD, Gonzalez-Lima F. Energy hypometabolism in posterior cingulate cortex of Alzheimer's patients: superficial laminar cytochrome oxidase associated with disease duration. $J$ Neurosci. 2001;21(13):4923-4930.

101. Valla J, Yaari R, Wolf AB, et al. Reduced posterior cingulate mitochondrial activity in expired young adult carriers of the APOE epsilon4 allele, the major late-onset Alzheimer's susceptibility gene. J Alzheimers Dis. 2010;22(1):307-313.

102. Shumake J, Gonzalez-Lima F. Brain systems underlying susceptibility to helplessness and depression. Behav Cogn Neurosci Rev. 2003;2(3):198-221.

103. Hamani C, Diwan M, Macedo CE, et al. Antidepressant-like effects of medial prefrontal cortex deep brain stimulation in rats. Biol Psychiatry. 2010;67(2):117-124

104. Michalikova S, Ennaceur A, van Rensburg R, Chazot PL. Emotional responses and memory performance of middle-aged CD1 mice in a 3D maze: effects of low infrared light. Neurobiol Learn Mem. 2008;89(4):480-488
Eye and Brain

\section{Publish your work in this journal}

Eye and Brain is an international, peer-reviewed, open access journa focusing on clinical and experimental research in the field of neuro-ophthalmology. All aspects of patient care are addressed within the journal as well as basic research. Papers covering original research, basic science, clinical and epidemiological studies, reviews and evaluations,

\section{Dovepress}

guidelines, expert opinion and commentary, case reports and extended reports are welcome. The manuscript management system is completely online and includes a very quick and fair peer-review system, which is all easy to use. Visit http://www.dovepress.com/testimonials.php to read real quotes from published authors. 